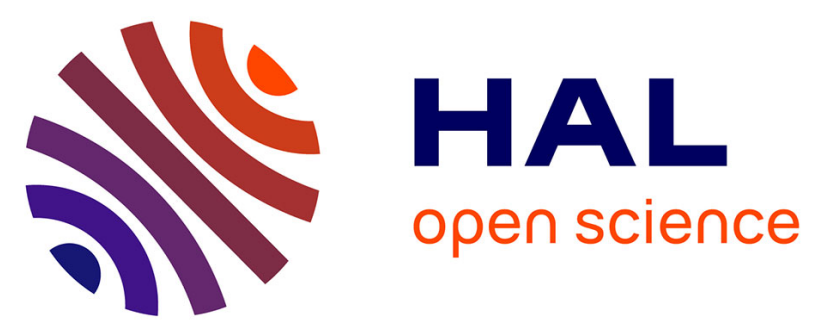

\title{
On the influence of boundary conditions when determining transport coefficients from finite samples of porous media. Assessment for tomographic images of real materials
}

Jianwei Shi, Germain Boyer, Valeri V. Mourzenko, Jean-François Thovert

\section{To cite this version:}

Jianwei Shi, Germain Boyer, Valeri V. Mourzenko, Jean-François Thovert. On the influence of boundary conditions when determining transport coefficients from finite samples of porous media. Assessment for tomographic images of real materials. Transport in Porous Media, 2020, 132, pp.561-590. 10.1007/s11242-020-01404-1 . hal-02501771

\section{HAL Id: hal-02501771 \\ https://hal.science/hal-02501771}

Submitted on 7 Mar 2020

HAL is a multi-disciplinary open access archive for the deposit and dissemination of scientific research documents, whether they are published or not. The documents may come from teaching and research institutions in France or abroad, or from public or private research centers.
L'archive ouverte pluridisciplinaire HAL, est destinée au dépôt et à la diffusion de documents scientifiques de niveau recherche, publiés ou non, émanant des établissements d'enseignement et de recherche français ou étrangers, des laboratoires publics ou privés. 


\title{
On the influence of boundary conditions when determining transport coefficients from finite samples of porous media. Assessment for tomographic images of real materials.
}

\author{
Jianwei Shi* and Germain Boyer \\ Institut de Radioprotection et de Sûreté Nucléaire (IRSN), PSN-RES, \\ SA2I, LIE, Cadarache, St Paul-lez-Durance, 13115, France \\ Valeri V. Mourzenko and Jean-François Thovert ${ }^{\dagger}$ \\ Institut P', CNRS - Université de Poitiers - ISAE-ENSMA, \\ 11 bd Marie et Pierre Curie, TSA 41123, 86073 POITIERS CEDEX 9, France
}

\begin{abstract}
The increasing access to $3 \mathrm{~d}$ digital images of porous media provides an ideal avenue for the determination of their transport properties, by solving the governing equations in their actual microscale geometry and evaluating the tensor coefficient that relates the mean flux and driving gradient. However, the first and puzzling question along the way is the choice of the conditions to be imposed for this resolution at the boundaries of the sample. This methodological issue is explored here with the purpose of quantifying the influence of the boundary conditions (BC) in relation with the parameters of the system (porosity, characteristic length scale of the microstructure, ratio of the phase conductivities), assessing the level of confidence associated with the predictions, devising criteria to anticipate the risk of serious artifacts, and if possible proposing ways to limit them. Although the terminology of thermal transfer is used, the developments apply to the upscaling of any transport property governed by a diffusion equation, including thermal or electrical conduction, mass diffusion or Darcy flow. Quantitative indicators are introduced for a rigorous individual or comparative assessment of conductivity tensors, and they are used in the analysis of the results of extensive calculations based on four tomographic images of various kinds of porous materials, with a broad range of conductivity contrasts, and various kinds of BC's. Ultimately, practical criteria are proposed for the a priori and a posteriori detection of at-risk situations, and a self-diagnosing protocol is proposed to screen out the influence of the BC's, when this is possible.
\end{abstract}

Keywords: Numerical upscaling; Conductivity; Permeability; Boundary condition; Tomography

\section{INTRODUCTION}

The present study was originally motivated by a practical problem, during the investigation of the thermal degradation of polymer-based composite materials in a fire safety context [29]. Since thermal conductivity is influential in the pyrolysis process and difficult to measure in degradated materials, it was decided to determine it on the basis of tomographic images at successive stages of the degradation. Direct solution of the Laplace equation in the exhaustively known microstructure is the natural avenue. But unsurprisingly, it was observed that the results can be very sensitive to the kind of boundary conditions (BC's) applied in the calculations. This is a well-known problem, commonly encountered in such situations. Therefore, it was thought of interest to investigate it in some details, in the hope to provide guidelines and criteria applicable in a wider context than our specific situation.

Since the earliest days of numerical upscaling based on tomographic digital images [30], various procedures based on different kinds of boundary conditions have been used and many examples taken from the literature are cited later in the text. Some of them such as $[10,32]$ implemented two or more procedures and briefly compare their results. The following review focuses on contributions which present more systematic

\footnotetext{
* Institut P', CNRS - Université de Poitiers - ISAE-ENSMA, 11 bd Marie et Pierre Curie, TSA 41123, 86073 POITIERS CEDEX 9, France

† jean.francois.thovert@univ-poitiers.fr
}

comparisons and/or address theoretical aspects of the issue. Note that a large part of the cited literature actually adresses the upscaling of Darcy flow in geological media, from a mesoscale permeability field to a macroscale permeability, rather than thermal conductivity, but the two problems are mathematically equivalent. Although a heat transfer terminology is used, the word "conductivity" can be understood throughout this paper as thermal, electric or hydraulic, i.e., permeability with temperature $T$ replaced by pressure $P$, or mass diffusivity (see Section II C).

The general review [28] of theoretical aspects of the upscaling techniques for Darcy flow in heterogeneneous media surweys various protocols and stresses the sensitivity of the determined block permeability to the BC's. Darcy flow upscaling procedures are also extensively reviewed in [11], including the aspects associated with BC's and post-treatments. Periodicity, permeameter and immersion conditions (see Section II E), as well as others involving the introduction of border regions (see below and Section II H) are surveyed, and their relative merits and shortcomings are discussed. More recently, various boundary conditions have been tested in [4] for the evaluation of the electrical conductivity of rock samples from tomographic images. It was stressed again that the choice of boundary conditions is important (with results differing by factors up to $3 / 2$ ), but no conclusion or advice was put forward.

In spite of their opposite denominations, the oversampling and undersampling approaches proceed from the same idea in the search of an intrinsic value of an effective conductivity. In the undersampling method, the sampling domain is reduced by excluding from the 
measurements a peripheral layer so that boundary effects are minimized. It is applied in [20], but without quantification of the difference it makes or investigation of the requirements for the removed layer thickness. The procedure described in Section II H proceeds along these lines. The oversampling approach follows the opposite way by using an enlarged sampling block so that the conductivity measured in the domain of interest is not "polluted" by the boundary effects confined in the peripheral region and becomes independent of the imposed boundary conditions. The term "oversampling" is due to [35] but the method was introduced earlier [13] and used under different names by others, such as [33]. It requires a knowledge of what surrounds the investigated domain, which can be available in the situations addressed in [13, 33, 35] where the aim is the coarsening of a detailed large scale permeability field, but not when operating with a tomographic image. However, the oversampling approach will be considered in a forthcoming study which extends the present work by making use of synthetic materials.

For completeness, let us mention that similar issues in the upscaling of the microscale Stokes equations for flows in porous media to obtain a macroscopic permeability tensor have also been treated. The problem is mathematically different, but sensitivity to the prescibed boundary conditions is also important, and even more critically that in a conduction process. Indeed, the solid phase is strictly impervious and the flow is confined in the pores, which corresponds to the limiting and most sensitive case of infinite conductivity contrasts in these situations explored in the present work. The classical BC's are of the same type as those for conduction problems, combining periodicity, Dirichlet (pressure) and Neumann (no flow) conditions. Several of them have been implemented in [25], yielding significantly different predictions, and the undersampling procedure was also used for the identification of a region that is not sensibly affected by the boundary conditions. Systematic investigations have also been conducted in [14], where many kinds of BC's have been applied and compare, including an effective medium approach similar to $[\mathrm{E}+\mathrm{P}]$ in the following (see Section II E).

Thus, it appears that a corpus of knowledge exists in the literature. Unfortunately, it consists in many comparisons of approaches in particular cases, and whereas the influence of the choice of a procedure and BC's is always pointed out, the differences between predictions is often presented in an illustrative and/or qualitative way. Even the most systematic studies provide general observations and mention some pitfalls but do not come up with practical recommendations. Quoting [11], it is not clear from previous work which of these approaches is the more accurate. It is likely that the method of choice will be case dependent. Thus, someone in search for practical advices for an application is at a loss to find explicit guidance.
Of course, at least in the case of tomographic sample images, there is no "right answer", since what lies beyond the sample boundaries is unknown. If two methods yield different results, it generally cannot be claimed that one of them is "right". However, it can sometimes be detected that one (or both) of them is wrong, with a quantitative estimates of just how wrong it has to be. Therefore, our work was conducted with several objectives in mind :

- contribute to the knowledge base by a systematic examination of a variety of materials, with quantification of the influence of the boundary conditions, in relation with the parameters of the system (volume fractions, characteristic length scale of the microstructure, contrast of the local conductivities);

- provide quantitative tools for the assessment of the expected level of confidence associated with predictions, and if possible, propose selfdiagnosing procedures;

- identify criteria (possibly just rules of thumb), a priori or a posteriori, for the detection of the risk-situations where serious artifacts can be expected and particular caution is required.

Fullfilling this involves extensive numerical calculations, but also the definition of quantitative indicators for a rigorous analysis of the results. The samples treated in the present work are tomographic images of several kinds of real materials. Phenomenological knowledge can be gained from this collection of particular cases, quantitative assessments are conducted for each of them, and general trends are identified. In order to explore systematically a wider range of morphological parameters, synthetic, numerically generated media are used in an ungoing extension which will be the object of another forthcoming paper.

The paper is organised as follows. Section II starts with a description of the context and of the investigated samples. The mathematical problem to be solved is then stated, and a brief description of the numerical solver is provided. The set of BC's considered in the study is introduced and some of their expected artefacts are commented. The procedure to obtain the full effective conductivity tensor is described, from the whole sample or from measurements in inner sub-domains (undersampling). Finally, some rigorous quantification tools for the comparative analysis of the results are introduced. The results are presented in Sections III-V, starting with global indicators such as the mean conductivities, the eigenvalues of the conductivity tensors and the distances between tensors resulting from different BC's. Then, qualitative comparisons in Section of the local fields are presented in IV, which provide a phenomenological picture of the effects of the boundary conditions, and a quantitative analysis of their differences is conducted. Finally, the undersampling approach is applied in Section V. A discussion in Section VI concludes the paper. 


\section{CONTEXT AND METHODS}

\section{A. Context}

As already mentioned, our initial motivation stemmed from a practical problem, for the determination on the basis of tomographic images of the thermal conductivity of thermally degraded polymer-based composite materials. The thermal conductivity of the constituents ranges from $\lambda_{g}=0.02$ to $0.2 \mathrm{~W} / \mathrm{m}$.K for the gas phase, depending on temperature and on the nature of the gas, from $\lambda_{s}=\mathrm{O}\left(10^{-1}\right)$ to $\mathrm{O}(1) \mathrm{W} / \mathrm{m} . \mathrm{K}$ for the polymer, and up to $20 \sim 30 \mathrm{~W} / \mathrm{m}$.K for alumina particles which are the main remaining solid constituent in the final stages of decomposition. Thus, the conductivity contrast can range from $\mathrm{O}(1)$ to $\mathrm{O}\left(10^{3}\right)$, with the solid generally more conducting than the gas phase.

Nevertheless, a much broader range was explored, with $10^{-4} \leq \lambda_{s} / \lambda_{g} \leq 10^{4}$. Its upper part is the usual situation in thermal conduction. However, the opposite case is worth considering since it is representative of other diffusive transports, governed by mathematically equivalent problems, such as electrical conduction or solute diffusion. Then, the interstitial fluid is much more conducting than the solid phase, which is nearly (if microporous) or totally impervious. Furthermore, the two phases are referred to as "pore" and "solid", but this bears no particular meaning since the developments apply for any mixture of two components with different conductivities. In particular, when considering Darcy flow in heterogenous media, what is called here "gas" and "solid" can correspond to regions with different and very contrasted permeabilities.

In the following, various kinds of media are considered, for a range of $\lambda_{s} / \lambda_{g}$, and the predictions of calculations with various kinds of boundary conditions are compared. Only binary media are considered, containing two phases with constant, isotropic conductivities. Media with continuous variations of the local conductivity could be treated in the same way. Some of the effects would probably be milder, but the general trends are not expected to differ and the methods and quantification tools presented in the following would retain their interest.

\section{B. Investigated materials}

The main characteristics of the investigated tomographic images are summarized in Table I. In all cases, the geometry is described by a phase function $Z$, defined in a $\left[L_{x} \times L_{y} \times L_{z}\right]$ array of cubic $p_{x}^{3}$ voxels, equal to 1 in the pores and to 0 in the solid. Two of its statistical moments are of a foremost interest. The first one is the porosity $\varepsilon$ which is the volume average $\langle Z\rangle$. For convenience, we sometimes speak in terms of the phase volume fractions $\varepsilon_{g}=\varepsilon$ and $\varepsilon_{s}=1-\varepsilon$. The second one is the spatial correlation function $R_{Z}(\mathbf{u})=$ $\operatorname{Covar}(Z(\mathbf{x}), Z(\mathbf{x}+\mathbf{u}))$ / $\operatorname{Var}(Z)$. The integral correlation length $l_{c}=\int_{0}^{\infty} R_{Z}(u) \mathrm{d} u$ (per direction, if $R_{Z}$ is anisotropic) provides a length scale associated with the microstructure. When $R_{Z}(\mathbf{u})$ happens to decay exponentially, this $l_{c}$ is also equal to the decay length.

Two kinds of porous media have been considered, starting with the thermally degraded polymer-based composite which initially motivated the present study, at two successive stages BP and WP of its degradation ( $\mathrm{P}$ for polymer, and $\mathrm{B}$ or $\mathrm{W}$ for black or white according to the sample color in each state). The samples have been prepared at C2MA (IMT Mines Alès) [12] and imaged at LEM3 (Lorraine University). BP has a roughly cylindrical shape, with its axis along the $z$ direction. Cross-sections at roughly mid-position normal to the $x$ and $z$-axis are shown in Fig. 1a,b. The material contains bubbles of various sizes, and it is obviously anisotropic. The large bubbles are markedly oblate, with their largest section roughly parallel to the $(x, y)$ plane. For the calculations, a parallelepipedic block of $[381 \times 401 \times 267]$ voxels was extracted. Bubbles with a broad range of sizes are also visible in the mid- $x$ and $z$ sections for the later stage WP in Fig. 1c,d. Due to the irregular sample shape, the computational domain was restricted to a $[85 \times 141 \times 83]$ block.

Then, two rock samples already examined in earlier works have been revisited. The first one is a Fontainebleau sandstone (FS), with porosity 0.0692, fully characterized in [31]. The $3 \mathrm{~d}$ image contains $[512]^{3}$ voxels (Fig.1e). The correlation function is isotropic and exponentially dacaying, with a decay length $l_{c}=4 p_{x}$. Thus, the sample size can be expressed as $\left[128 l_{c}\right]^{3}$. The second one is a Bentheim sandstone (BS), with porosity 0.232 , studied in [32]. Again, the correlation function is isotropic and exponential, with a decay length $l_{c}=6.5 p_{x}$. The $3 \mathrm{~d}$ image contains $[500]^{3}$ voxels (Fig.1f), which can be expressed as $\left[77 l_{c}\right]^{3}$.

\section{Local and upscaled formulations}

Stationary thermal conduction in a heterogeneous medium with position-dependent thermal conductivity $\lambda$ is governed on the local scale by Fourier's law and a conservation equation

$$
\mathbf{q}=-\lambda \nabla T, \quad \nabla \cdot \mathbf{q}=0
$$

where $\mathbf{q}$ is the heat flux and $T$ is the temperature. However, if the medium statististical properties are spatially invariant, it can be regarded on a larger scale as an equivalent homogeneous material with effective properties, and in particular with an effective conductivity $\Lambda$ which relates the locally averaged flux and gradient

$$
\langle\mathbf{q}\rangle=-\boldsymbol{\Lambda} \cdot\langle\nabla T\rangle, \quad \nabla \cdot\langle\mathbf{q}\rangle=0
$$

Even though the local conductivity is assumed to be isotropic, quantified by a scalar coefficient $\lambda$, the effective coefficient $\boldsymbol{\Lambda}$ is in general tensorial, since the medium structure can be anisotropic. It may be that the medium is not strictly statistically homogeneous, but that its characteristics are slowly varying. Then, 


\begin{tabular}{lcccl} 
Sample & $\begin{array}{c}p_{x} \\
(\mu \mathrm{m})\end{array}$ & $\begin{array}{c}\text { Block size } \\
{\left[L_{x} \times L_{y} \times L_{z}\right]}\end{array}$ & $\varepsilon$ & Physical length scale \\
\hline BP & 9.8 & $381 \times 401 \times 267$ & 0.280 & Integral lengths $l_{c x} \approx 22 p_{x}, l_{c y} \approx 22 p_{x}, l_{c z} \approx 5 p_{x}$ \\
WP & 9.8 & $85 \times 141 \times 83$ & 0.599 & Integral lengths $l_{c x} \approx 3.6 p_{x}, l_{c y} \approx 11 p_{x}, l_{c z} \approx 9 p_{x}$ \\
FS & 6.3 & {$[512]^{3} \approx\left[128 l_{c}\right]^{3}$} & 0.0692 & Correlation exponential decay length, $l_{c}=4 p_{x}$ \\
BS & 6.0 & {$[500]^{3} \approx\left[77 l_{c}\right]^{3}$} & 0.232 & Correlation exponential decay length, $l_{c}=6.5 p_{x}$ \\
\hline
\end{tabular}

TABLE I. Main sample characteristics : voxel size $p_{x}$, computational domain size $\left[L_{x} \times L_{y} \times L_{z}\right]$ in voxels, porosity $\varepsilon$, and a typical scale for their microstructure.

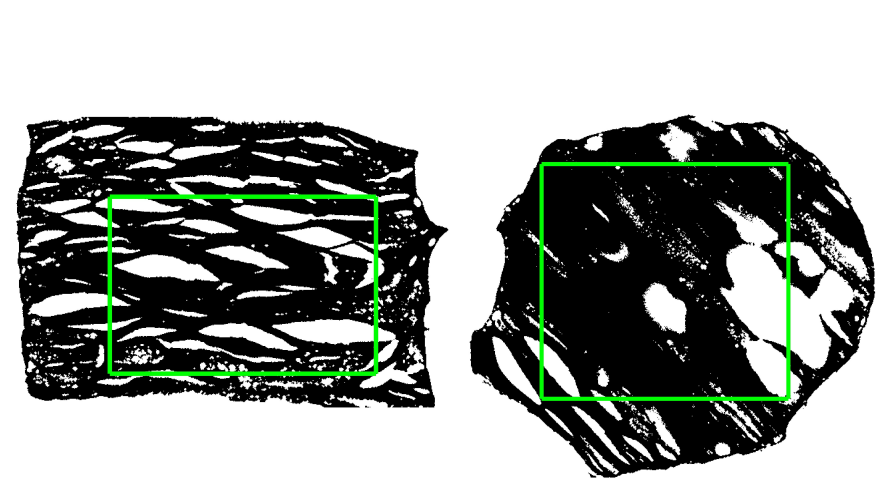

(a)

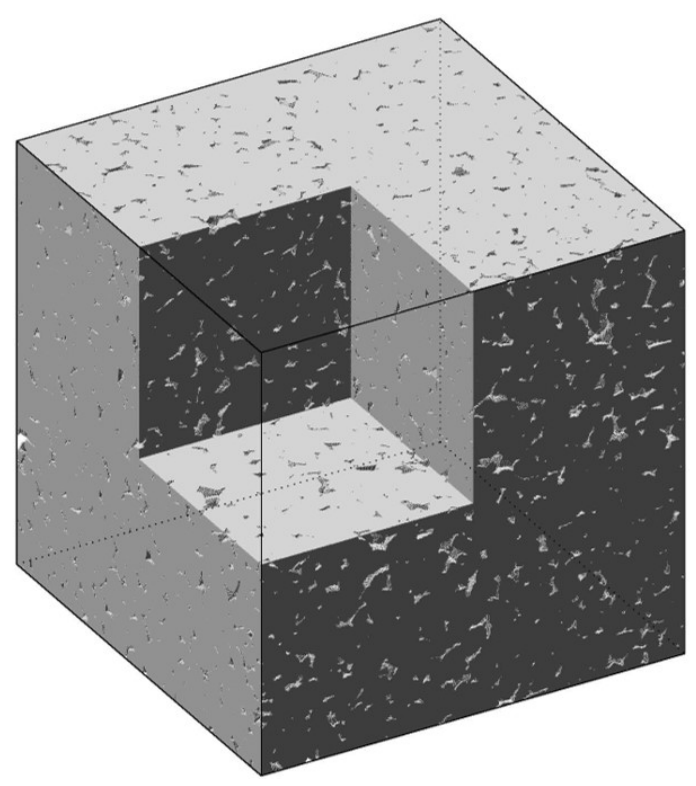

(e) (b)

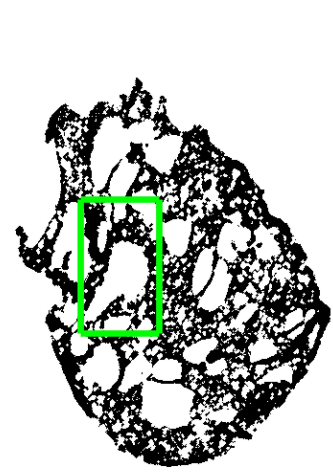

(c)

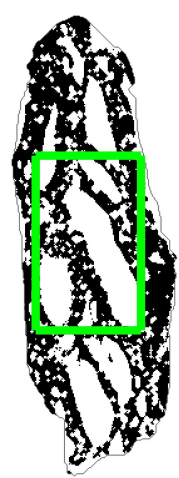

(d)

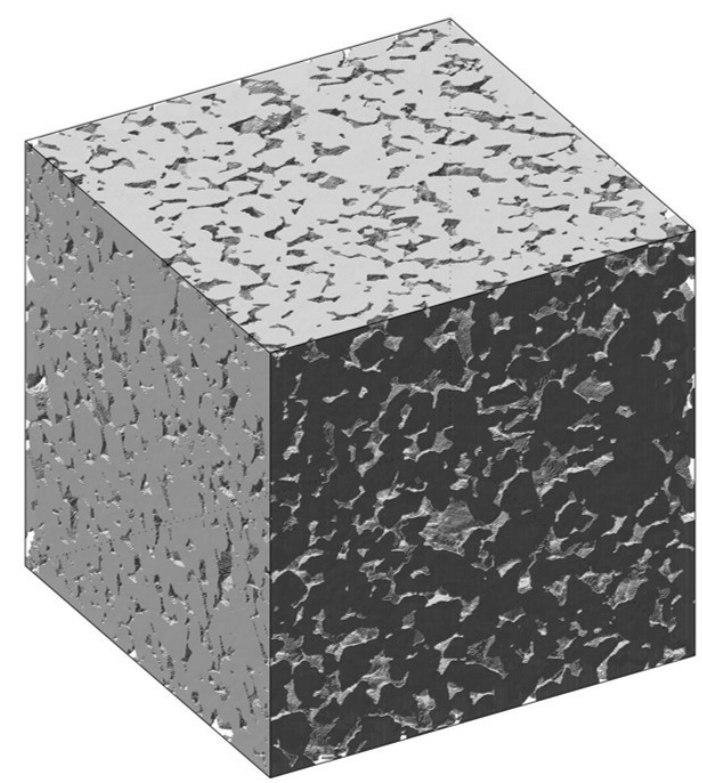

(f)

FIG. 1. Mid-sections through sample BP, normal to the $x$ (a) and $z$ (b) axes. Mid-section through sample WP, normal to the $x$ (c) and $z$ (d) axes. Samples FS (e) and BS (f). The green frames in (a-d) show the computational domain cut from the whole image.

(2) still applies with a position-dependent $\boldsymbol{\Lambda}$ if the macroscale of the regional variations is much larger than the microscale of the $\lambda$ fluctuations. Then, an intermediate scale (the so-called Representative Elementary Volume) smaller than the former and larger than the latter can exist, upon which volume averages of the flux and gradient can be taken. Note that the REV is often defined only in geometrical terms, as the minimal averaging volume required to damp porosity fluctuations, whereas we are talking here of the averaging volume necessary for a robust estimation of a transport coefficient. Very often, this is a more stringent criterion, for instance close to a percolation threshold when the conductivity contrast is strong. But as illustrated in Section $\mathrm{V}$, it can also be a milder criterion in the case of a moderate conductivity contrast or if the most present phase is also the most conducting.

The interest of the upscaling from (1) to (2) is of course in the tremendeously reduced computational effort required for simulations, since much coarser volume elements with effective properties can be used when solving (2). The theoretical background for the 
homogenization process is classical and not detailed here (see e.g., [2]). Let us just mention that $\boldsymbol{\Lambda}$ is a symmetric, positive tensor.

The following developments apply to many other processes, such as mass diffusion of electrical conduction, which give rise to similar problems and which can be handled in the same way. Furthermore, even though creeping fluid flow through a porous medium is governed locally by Stokes equations, a first upscaling step can reduce the flow problem into a homogenized form similar to (1) involving Darcy's law,

$$
\overline{\mathbf{v}}=-\frac{1}{\mu} k \nabla p, \quad \nabla \cdot \overline{\mathbf{v}}=0
$$

where $\overline{\mathbf{v}}$ is the seepage velocity, $p$ is the pressure, $\mu$ is the fluid viscosity and $k$ is a permeability coefficient. This formulation applies on a scale much larger than the medium microstructure, but the medium can be heretogeneous on a still larger scale which makes $k$ position-dependent. In this case, a second upscaling can be performed to obtain a counterpart of (2) involving an effective permeability tensor $\mathbf{K}$.

\section{Solution of the local problem}

The thermal problem governed by (1) is solved in a domain $\Omega$ subject to various conditions on its boundary, which are detailed in the next Section IIE and which all consist in imposing temperatures or temperature jumps on $\partial \Omega$. The gas phase is given a unit conductivity $\lambda_{g}=1$ and the solid conductivity is set in a range $\lambda_{s}=10^{-4}$ to $10^{4}$.

The solver is a distant descendant of that presented in [17], where the formulation is described in full details. Equations (1) are discretized in a finite volume formulation, according to the so-called box integration method. The temperature is determined at the vertices of the cubic volume elements. The resulting set of linear equations $\mathbf{A} \cdot \mathbf{T}=\mathbf{B}$ is solved by a conjugate gradient method. Iterations are stopped when the global relative residue $\|\mathbf{A} \cdot \mathbf{T}-\mathbf{B}\|$ becomes smaller than $10^{-6}$ $\|\mathbf{B}\|$. Note that this a much more stringent stopping criterion than for routine applications, where $10^{-4}$ is regarded as sufficient. This much finer (and numerically much costlier) accuracy is required for a reliable comparison of the solutions for different BC's. The $10^{-6}$ criterion for the global residue translates into a $10^{-4}$ relative accuracy for the components of the predicted mean flux, and to at most $10^{-3}$ for pointwise temperature, relative to the overall temperature drop.

The volume average $\langle\mathbf{q}\rangle$ of the flux mean in $\Omega$ is actually evaluated by the surface integral

$$
\langle\mathbf{q}\rangle=\frac{1}{\Omega} \int_{\partial \Omega} \mathbf{q}(\mathbf{x}) \cdot \mathbf{n} \mathbf{x} \mathrm{d} s
$$

where $\mathbf{n}$ is the unit outwards vector normal to $\partial \Omega$. Note that the volume average of the gradient $\langle\nabla T\rangle$ can also be obtained by the a surface integral

$$
\langle\nabla T\rangle=\frac{1}{\Omega} \int_{\partial \Omega} T \mathbf{n} \mathrm{d} s
$$

\section{E. Investigated boundary conditions}

When faced with a finite sample $\Omega$ without knowledge of what lies beyond its boundaries $\partial \Omega$, a natural way to set the overall boundary contitions for the solution of problem (1) is to try and mimic real or virtual experimental settings. Permeameter conditions, nammed after a common apparatus for the measurement of permeability, correspond to the situation where the sample is placed between two isopotential chambers (Dirichlet pressure conditions, or temperature in the present terms) and enclosed transversally in an impervious jacket. One may imagine a virtual experiment where the single jacketed sample is replaced by an infinite layer of juxtaposed replicas exposed to the same upstream and downstream Dirichlet conditions. This correspond to $[\mathrm{D} / \mathrm{P}]$ as defined below. One may also imagine that the entire space is covered by such identical replicas, which gives rise to $[\mathrm{P} / \mathrm{P}]$ below. One may consider that the sample is encased in an homogeneous material with the same effective conductivity (to be determined) and solve the problem in an enlarged domain with periodicity conditions, which corresponds to $[\mathrm{E}+\mathrm{P}]$. Finally one may assume that the far-field conditions of $[\mathrm{E}+\mathrm{P}]$ apply down to $\partial \Omega$ and impose there Dirichlet conditions corresponding to a prescribed mean gradient. These so-called "Immersion" conditions are denoted [D/D] below. In the labels $[\mathrm{X} / \mathrm{Y}], \mathrm{X}$ and $\mathrm{Y}$ stand for the axial and transverse conditions, respectively, with $\mathrm{P}$ for periodicity and $\mathrm{D}$ for Dirichlet. $[\mathrm{E}+\mathrm{P}]$ stands for "encased" with full periodicity.

In the present investigations, the following four kinds of conditions have been used for the solution of problem (1), imposed on the boundaries of the computational domain $\partial \Omega$ or $\partial \Omega^{+}$.

[P/P] Periodicity of fluxes and temperature gradient is applied, regardless of whether the medium structure is periodic or not. If $\mathbf{x}_{1}$ and $\mathbf{x}_{2}$ are homologous points on opposite faces of the domain boundaries

$$
\mathbf{q}_{2}=\mathbf{q}_{1}, \quad T_{2}-T_{1}=\mathbf{G} \cdot\left(\mathbf{x}_{2}-\mathbf{x}_{1}\right)
$$

where $\mathbf{G}$ is a prescribed macroscopic temperature gradient.

[D/P] Dirichlet conditions are imposed on inlet/outlet faces, while periodicity is kept in the transerve directions. For instance, for a calculation along the $x$ direction, 


$$
T(x=0)=G L_{x} \quad T\left(x=L_{x}\right)=0
$$

$$
\mathbf{q}_{2}=\mathbf{q}_{1}, \quad T_{2}=T_{1} \text { at homologous points }\left(\mathbf{x}_{1}, \mathbf{x}_{2}\right) \text { on opposite transverse faces }
$$

[D/D] Immersion conditions are imposed, whereby temperature is prescribed on the entire boundary $\partial \Omega$ of the domain, with

$$
T(\mathbf{x})=\mathbf{G} \cdot \mathbf{x}, \quad \text { on } \partial \Omega
$$

within some arbitrary additive constant. In practice, with $\mathbf{G}$ along one of the axes of coordinates, this results in Dirichlet conditions (7a) on inlet/outlet faces and linear profiles along the transverse faces.

[E+P] Embedding the sample $\Omega$ in a sheath of homogeneous material with appropriate properties is an attempt to screen out most of the influence of the outer BC's. Thus, the problem is solved in a wider domain $\Omega^{+} \supset \Omega$, with periodicity conditions (6) applied on $\partial \Omega^{+}$. Integrations $(4,5)$ to obtain the mean flux and gradient are restricted to $\Omega$. The embedding medium is given an conductivity $\boldsymbol{\Lambda}_{E}$, possibly anisotropic, equal to that obtained in $\Omega$. Since the latter is not known beforehand, this is an iterative process. A reasonable guess for $\boldsymbol{\Lambda}_{E}$ is used first, which is updated after successive resolutions, until convergence. The layer added on the six faces of the samples was 16 voxels thick. It has been checked that this is sufficient to make the results nearly independent of the layer thickness, both for the global parameters and for the local deviations in $\Omega$, by a systematic comparison with data obtained with a thickness of 8 voxels.

Examples of applications can be found in $[4,10,11$, $18,30]$ for $[\mathrm{P} / \mathrm{P}]$, in $[3,7,32]$ for $[\mathrm{D} / \mathrm{P}]$ and in $[6,11,14,21,26]$ for $[D / D]$. Although the idea of a self-consistent scheme is very common in theoretical models for the conductivity of composites such as those of Bruggeman [8] or Landauer [19], it seems that its numerical counterpart $[\mathrm{E}+\mathrm{P}]$ has not been implemented in earlier works for the upscaling of conduction properties (but it is in [14] for the upscaling of the Stokes flow equations).

Other kinds of BC's are found in the literature which are not considered here. As already mentioned, the so-called permeameter conditions are used quite often [3, 5, 9-11, 16, 20, 22-24, 31]. Their no-flux condition through the transverse boundaries obviously constrains the flux directionally and cannot provide reliable estimates of the transverse components of $\langle\mathbf{q}\rangle$. Thus, this approach is not appropriate for the determination of the full tensors $\boldsymbol{\Lambda}$ or $\mathbf{K}$ in anisotropic media, and it was not considered for this reason. In other cases, periodicity conditions $[\mathrm{P} / \mathrm{P}]$ are applied with a sample made periodic by juxtaposing mirrored images of $\Omega[4,14]$. Again, this introduces directional constraints and the eigen-directions of $\boldsymbol{\Lambda}$ or $\mathbf{K}$ can only be found aligned with the artificial planes of symmetry. Various ad-hoc methods can be found where [P/P] is applied to $\Omega$ supplemented with upstream and/or downstream layers of a homogeneous material, in order ensure the connection of the conductive paths on the inlet and outlet sides of $\Omega[3,30]$. Finally, a counterpart of [D/D] was considered in [26] where the normal flux instead of the temperature is prescribed on the boundaries,

$$
\mathbf{q}(\mathbf{x}) \cdot \mathbf{n}=\mathbf{B} \cdot \mathbf{n}, \quad \text { on } \partial \Omega
$$

where $\mathbf{B}$ is a prescribed vector, which (4) shows to correspond to $\langle\mathbf{q}\rangle$.

\section{F. Artefacts associated with the boundary conditions}

With the exception of $[\mathrm{P} / \mathrm{P}]$ applied to periodic media, which generally means model media, all the conditions listed in Sec.IIE present some undesirable features. Consider for illustration purposes the most severe situations where only the pore phase is conducting $\left(\lambda_{s}=0\right)$. The same effects, though milder, are expected for smaller contrasts.

Periodicity $[\mathrm{P} / \mathrm{P}]$ imposes that the fluxes on opposite faces are equal, but since the phase arrangements in these faces do not match in aperiodic media, the flux has to cross a plane with a much reduced open fraction, equal to $\varepsilon^{2}$ in the average (Fig. 2a). This introduces a "skin" resistance, and the overall conductivity is underestimated. With Dirichlet conditions [D/P], flux can enter any pore showing on the inlet face of $\Omega$, although some of them are actually dead-ends and would receive no flux from the actual upstream material (Fig. $2 b)$. The overall conductivity is overestimated. With the pressure condition (8) of [D/D], flux can leave or enter any pore showing on a lateral face of $\Omega$, as if some continuous conducting material lay beyond $\partial \Omega$. This creates long-range connections all along the lateral faces (Fig. 2c), which can behave as an apparent lateral conducting skin. The overall conductivity is overestimated.

Note that the artefacts associated with $[\mathrm{P} / \mathrm{P}]$ and $[\mathrm{D} / \mathrm{P}]$ are local features. They affect the transfers through a surface which the flux has to cross (if $\perp$ $\langle\nabla T\rangle$ ) or might cross (if $\|\langle\nabla T\rangle$ ). Conversely, the artefact introduced by $[\mathrm{D} / \mathrm{D}]$ has long range effects generally with stronger impact on the predicted conductivity, as indeed observed in the following. In a caricatured situation, it would yield non-zero flux and effective conductivity if $\Omega$ were entirely filled with insulat- 
ing material except for small unconnected conducting parts located at its corners.

\section{G. Determination of a full tensor $\boldsymbol{\Lambda}$}

Equations (1) are solved subject to one of the BC's listed in Sec.II G, say [BC], with the vector $\mathbf{G}$ set successively along the $x$-, $y$ - and $z$-directions. In each case, the mean flux $\langle\boldsymbol{q}\rangle_{\xi}$ and gradient $\langle\nabla T\rangle_{\xi}(\xi=x, y$ or $z)$ are evaluated by means of $(4,5)$. Note that $\langle\nabla T\rangle$ in $\Omega$ is equal to $\mathbf{G}$ in the cases of $[\mathrm{P} / \mathrm{P}],[\mathrm{D} / \mathrm{P}]$ and $[\mathrm{D} / \mathrm{D}]$, as shown by injecting (6), (7) or (8) in (5). However, when $\mathbf{G}$ is applied on the boundaries of the enlarged domain $\Omega^{+}$for conditions [E+P], the mean gradient in $\Omega$ can be different and has to be calculated from the temperature field by application of (5). Then, the following set of 9 linear equations is solved to determined the 9 components of $\Lambda_{[B C]}$

$$
\langle\mathbf{q}\rangle_{\xi}=-\boldsymbol{\Lambda}_{[B C]} \cdot\langle\nabla T\rangle_{\xi}, \quad \xi=x, y \text { and } z
$$

With conditions $[\mathrm{P} / \mathrm{P}],[\mathrm{D} / \mathrm{D}]$ and $[\mathrm{E}+\mathrm{P}]$, the solutions obtained with different vectors $\mathbf{G}$ can be superposed, in view of the linearity of the governing equations. Therefore, the solution for any $\mathbf{G}$ can be obtained by a linear combination of the solutions with $\mathbf{G}$ set along the three axes, and $\boldsymbol{\Lambda}_{[B C]}$ determined by (10) can be used to predict the mean flux resulting from any mean gradient consistent with the specific conditions [BC]. This does not mean of course that identical mean fluxes result from identical mean gradients if the $T$-fields are constrained by different conditions (6) or (8), for instance.

Conversely, the solutions obtained with [D/P] applied along $x, y$ and $z$ cannot be superposed. Thus, the derivation of $\boldsymbol{\Lambda}_{[D / P]}$ is only formal, and (10) applies only for $\mathbf{G}$ set along $x, y$ or $z$. Anyway, it is difficult to conceive a numerical experiment where conditions of the type $[\mathrm{D} / \mathrm{P}]$ would be imposed along a direction oblique relative to a parallelepipedic sample. Note that the same observations apply as well to the permeameter conditions where transverse periodicity is replaced by a no-flux condition. However, this does not preclude $\boldsymbol{\Lambda}_{[D / P]}$ from bearing information about the sample conductive properties.

The effective tensor $\boldsymbol{\Lambda}$ should be symmetric, and $\boldsymbol{\Lambda}_{[P / P]}$ obtained by (10) with periodicity conditions $[\mathrm{P} / \mathrm{P}]$ is indeed symmetric. The demonstration relies on a reciprocal theorem (see e.g., [2]). The same line of reasoning shows that $\Lambda_{[D / D]}$ is also symmetric. Interestingly, this also applies to the flux immersion (9) [26]. However, $\boldsymbol{\Lambda}_{[D / P]}$ and $\boldsymbol{\Lambda}_{[E+P]}$ are not necessarily symmetric, even though periodicity conditions are applied on $\partial \Omega^{+}$in the case of $[\mathrm{E}+\mathrm{P}]$. Symmetry would be ensured with $[\mathrm{E}+\mathrm{P}]$ only if the averages used in (10) were evaluated over $\partial \Omega^{+}$instead of $\partial \Omega$.

Asymmetric macroscopic tensors are defective, and two techniques are widely used to put up with situations where the upscaling procedure yields an asymmetric result. The simplest one is to make the tensor symmetric by averaging it with its transpose,

$$
\Lambda=\left(\boldsymbol{\Lambda}_{[B C]}+\boldsymbol{\Lambda}_{[B C]}^{t}\right) / 2
$$

Another approach is to supplement (10) with additional equations stating the symmetry of $\boldsymbol{\Lambda}_{[B C]}$. The system becomes overdetermined, and it has to be solved in some least-square sense. Generally, some residual asymmetry remains which can be eliminated with (11) [11]. Such expedients are necessary for the practical use of $\boldsymbol{\Lambda}$ in simulation models, but no such step was taken in the present investigation. The purpose is not to cure the asymmetry of $\boldsymbol{\Lambda}_{B C C}$, but to quantify it and explore the circumstances of its occurence.

\section{H. Undersampling}

The fundamental idea underlying the application of upscaled models is the belief that an effective coefficient $\boldsymbol{\Lambda}$ exists which relates the locally averaged flux and gradient, regardless of the circumstances (i.e., farfield conditions) which induce $\langle\nabla T\rangle$ at the position where (2) is applied. On this premise, it can be attempted to determine this value of $\Lambda$ by focusing on some inner subdomain $\Omega_{c}$ in $\Omega$. If the tensor $\Lambda_{c}$ obtained by (10) with the mean fluxes and gradients given by the integrals $(4,5)$ applied to $\Omega_{c}$ is found independent of the conditions applied at $\partial \Omega$, this $\Lambda$ fulfills the aforementioned requirement for its use in upscaled models. This methodology has been applied in [20] for conduction processes, and in [25] for fluid flow.

In practice, we have applied this approach with subdomains $\Omega_{c}(M)$ obtained by removing from $\Omega$ a peripheral layer with thickness $M$. The conductivity tensor of $\Omega_{c}$ obtained from the solution of (1) with conditions [BC] at $\partial \Omega$ is denoted $\Lambda_{[B C], c}(M)$.

Obviously, a thick enough margin ( $M$ not too small) is required to screen out the influence of the outer conditions. On the other hand, the subvolume $\Omega_{c}$ has to be sufficient ( $M$ not too large) to remain representative, i.e., to prevent the occurence of large statistical fluctuations. If the whole sample $\Omega$ is large enough, an intermediate range for $M$ can exist where both of these criteria are fulfilled. This means that over this interval, $\boldsymbol{\Lambda}_{[B C], c}(M)$ should be independent of [BC] (first criterion) and of $M$ (second criterion). This constant value would be the effective tensor $\boldsymbol{\Lambda}$ for the investigated material.

Of course, the terms "constant" and "independent" have to be understood within some practical tolerance, and a quantitative indicator is required to measure the difference between two tensors. Such an indicator is defined in Sec.II I.

\section{Notations and quantification tools}

A few notations and definitions are introduced here, which are used in the subsequent discussions. First, the 


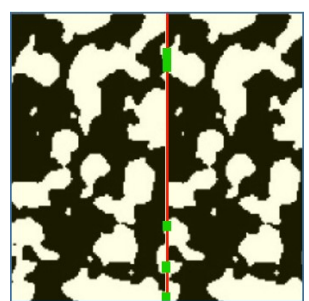

(a)

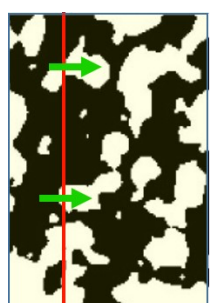

(b)

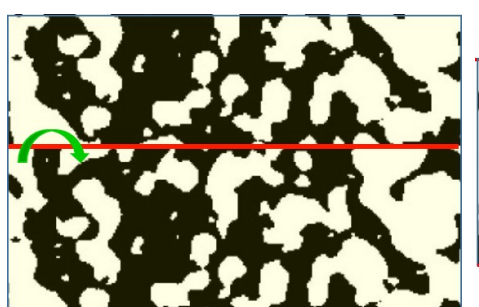

(c)

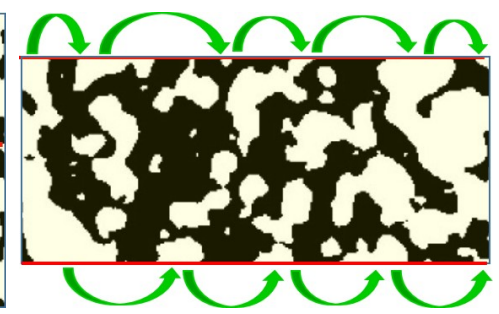

(d)

FIG. 2. Artefacts associated with the $[\mathrm{P} / \mathrm{P}],[\mathrm{D} / \mathrm{P}]$ and $[\mathrm{D} / \mathrm{D}]$. Examples when only the white phase (pores) is conducting. The mean flux is oriented from left to right in all cases. (a) With $[\mathrm{P} / \mathrm{P}]$, the inlet and outlet faces have a reduced open fraction (in green) due to geometrical mismatch. (b) With [D/P] and [D/D], flux enters all open parts of the inlet face, although some are actually dead-ends (arrows; simil at the outlet). (c) At transverse boundaries, $[\mathrm{P} / \mathrm{P}]$ and $[\mathrm{D} / \mathrm{P}]$ can create links between unconnected pores (arrow), or ignore links which should exist, whereas (d) [D/D] creates long-range connections between all pores showing on a face.

mean of the diagonal terms of a tensor $\Lambda$ is denoted $\bar{\Lambda}$ and an anisotropy index $\mathscr{N}$ is defined as the ratio of its largest and smallest eigenvalues, $\Lambda_{\operatorname{Max}}$ and $\Lambda_{\text {Min }}$,

$$
\bar{\Lambda}=\frac{1}{3} \sum_{i} \Lambda_{i i}, \quad \mathscr{N}=\frac{\Lambda_{\text {Max }}}{\Lambda_{\text {Min }}}
$$

A distance is introduced to quantify the difference between two tensors, say $\boldsymbol{\Lambda}_{1}$ and $\boldsymbol{\Lambda}_{2}$, resulting for in- stance from different upscaling protocols. In response to a same unit gradient $\boldsymbol{g}$, these tensors predict fluxes $\boldsymbol{q}_{i}=-\boldsymbol{\Lambda}_{i} \cdot \boldsymbol{g}$. The squared norm of their deviation $\left\|\boldsymbol{q}_{2}-\boldsymbol{q}_{1}\right\|^{2}$ is equal to $\boldsymbol{g}^{t} \cdot\left(\boldsymbol{\Lambda}_{2}-\boldsymbol{\Lambda}_{1}\right)^{t} \cdot\left(\boldsymbol{\Lambda}_{2}-\boldsymbol{\Lambda}_{1}\right) \cdot \boldsymbol{g}$. It is maximum when $\boldsymbol{g}$ is aligned with the eigendirection of $\left(\boldsymbol{\Lambda}_{2}-\boldsymbol{\Lambda}_{1}\right)^{t} \cdot\left(\boldsymbol{\Lambda}_{2}-\boldsymbol{\Lambda}_{1}\right)$ associated with its largest eigenvalue. Therefore, we mreasure the difference of $\boldsymbol{\Lambda}_{1}$ and $\boldsymbol{\Lambda}_{2}$ by the distance $\mathscr{D}$ and the normalized dimensionless quantity $\mathscr{D}^{\prime}$ defined by

$$
\mathscr{D}^{2}\left(\boldsymbol{\Lambda}_{1}, \boldsymbol{\Lambda}_{2}\right)=\text { largest eigenvalue of }\left[\left(\boldsymbol{\Lambda}_{2}-\boldsymbol{\Lambda}_{1}\right)^{t} \cdot\left(\boldsymbol{\Lambda}_{2}-\boldsymbol{\Lambda}_{1}\right)\right], \quad \mathscr{D}^{\prime}=\frac{\mathscr{D}}{\left[\overline{\Lambda_{1}} \overline{\Lambda_{2}}\right]^{1 / 2}}
$$

Thus, $\mathscr{D}^{\prime}\left(\boldsymbol{\Lambda}_{1}, \boldsymbol{\Lambda}_{2}\right)$ is the maximal relative deviation (combining magnitude and direction differences) of the fluxes predicted by $\boldsymbol{\Lambda}_{1}$ and $\boldsymbol{\Lambda}_{2}$ when applied to the same gradient. Note that the $\Lambda_{i}$ 's do not need to be symmetric in the definition (13), and that $\mathscr{D}$ is a distance in the mathematical sense, i.e., a symmetric positive-definite function satisfying the triangle inequality. The distance $\mathscr{D}$ actually derives from the matrix norm $\|\boldsymbol{A}\|^{2}=$ spectral radius of $\boldsymbol{A}^{t} \cdot \boldsymbol{A}$, with
$\mathscr{D}\left(\boldsymbol{\Lambda}_{1}, \boldsymbol{\Lambda}_{2}\right)=\left\|\boldsymbol{\Lambda}_{1}-\boldsymbol{\Lambda}_{2}\right\|$. Although $\mathscr{D}$ is used in other contexts to measure the difference between matrices (to monitor the convergence of iterative numerical schemes [27]) we are not aware of its use for the comparison of tensorial transport coefficients in earlier works. Finally, an additional quantity of interest is the asymmetry index $\mathscr{A}$ and its normalized dimensionless counterpart $\mathscr{A}^{\prime}$ defined by

$$
\mathscr{A}^{2}(\boldsymbol{\Lambda})=\sum_{1 \leq i<j \leq 3}\left[\left(\Lambda_{i j}-\Lambda_{j i}\right) / 2\right]^{2}, \quad \mathscr{A}^{\prime}(\boldsymbol{\Lambda})=\mathscr{A}(\boldsymbol{\Lambda}) / \bar{\Lambda}
$$

This indicator has an interesting relation with $\mathscr{D}$, namely that if $\boldsymbol{\Lambda}_{2}-\boldsymbol{\Lambda}_{1}$ is antisymmetric, then $\mathscr{D}\left(\boldsymbol{\Lambda}_{1}, \boldsymbol{\Lambda}_{2}\right)=\mathscr{A}\left(\boldsymbol{\Lambda}_{2}-\boldsymbol{\Lambda}_{1}\right)$. This implies that if a tensor $\Lambda_{[B C]}$ resulting from an upscaling procedure is not symmetric, it differs from $\boldsymbol{\Lambda}$ obtained by the symmetrization technique (11) by $\mathscr{A}\left(\Lambda_{[B C]}\right)$, and by at least that much from any possible acceptable (and therefore symmetric) conductivity tensor. Of course, this is only a lower bound for the error. For instance, if $\boldsymbol{\Lambda}_{[B C]}$ differs for all its components from an actual spherical tensor $\Lambda \boldsymbol{I}$ by independent random gaussian errors with standard deviation $\sigma \Lambda$, then
$\left\langle\mathscr{A}^{\prime}\left(\boldsymbol{\Lambda}_{[B C]}\right)\right\rangle \approx 0.33 \sigma$ and $\left\langle\mathscr{D}^{\prime}\left(\boldsymbol{\Lambda}_{[B C]}, \Lambda \boldsymbol{I}\right)\right\rangle \approx 1.6 \sigma \approx$ $4.9\left\langle\mathscr{A}^{\prime}\right\rangle$ (averages over $10^{8}$ Monte Carlo realizations).

Finally, the arithmetic and harmonic volume averages of the local conductivities are denoted by $\langle\lambda\rangle$ and $\langle\lambda\rangle_{H}$. They correspond to the fully general upper and lower Wiener's bounds [34]. In addition, Hashin \& Shtrikman's upper and lower bounds [15] are denoted by $\Lambda_{H S}^{U}$ and $\Lambda_{H S}^{L}$. Both sets of bounds only depend on the phase conductivities and volume fractions, but the tighter Hashin \& Shtrikman's bounds apply only to isotropic media. 


\section{RESULTS FOR GLOBAL INDICATORS}

We consider here some global indicators, resulting from the solution of problem (1) subject to the boundary condictions $[\mathrm{P} / \mathrm{P}],[\mathrm{D} / \mathrm{P}],[\mathrm{D} / \mathrm{D}]$ and $[\mathrm{E}+\mathrm{P}]$, with a broad range of conductivity contrasts $10^{-4} \leq \lambda_{s} / \lambda_{g} \leq$ $10^{4}$.

The simplest indicator and the first considered here is the mean conductivity $\bar{\Lambda}$. However, since some of the samples are anisotropic, the eigenvalues $\Lambda_{\operatorname{Max}}$ and $\Lambda_{\text {Min }}$ of $\boldsymbol{\Lambda}$ are also addressed. They are plotted for the polymer-based materials BP and WP in Figs.3 and 4, as functions $\lambda_{s} / \lambda_{g}$. For an easier comparison, they are normalized by the volume average $\langle\lambda\rangle$. Note that the ratio $\bar{\Lambda} /\langle\lambda\rangle$ can be viewed as a tortuosity factor, since it approaches one when most heat flow takes place along straight streamlines in the most conducting phase. Several general features stand out, whatever the BC's.

(a) When the conductivity contrast is very large, one expects that one of the phases ultimately plays a negligible role, and that an infinite ratio or some very large finite value makes vanishing difference. This is confirmed in all cases, with established asymptotical values beyond $10^{ \pm 3}$, except when the solid tends to become insulating in BP. It has been checked that the gas space is percolating, which implies that some non-zero asymptotic value exists when $\lambda_{s} / \lambda_{g} \rightarrow 0$, but convergence is not reached in the investigated range.

(b) For comparison, Wiener's lower bound $\langle\lambda\rangle_{H}$ and Hashin \& Shtrikman's bounds are also plotted in Figs.3,4 (Wiener's upper bound corresponds to unity). Of course, all these bounds provide reasonable estimates for small contrasts, but they grossly deviate from the numerical data for $\lambda_{s} / \lambda_{g}$ beyond $10^{ \pm 1}$. Note that values larger than $\Lambda_{H S}^{U}$ (Fig.3a) or smaller than $\Lambda_{H S}^{L}$ (Fig.3c) exist. This is not aberrant, since Hashin \& Shtrikman's bounds apply to isotropic media and do not constrain $\Lambda_{\text {Max }}$ and $\Lambda_{\text {Min }}$ in anisotropic media.

(c) Sample BP is strongly anisotropic. The anisotropy index $\mathscr{N}$ is of the order of 20 when $\lambda_{s} \ll \lambda_{g}$ and 2 when $\lambda_{s} \gg \lambda_{g}$. Sample WP is also anisotropic but in a lesser respect, with $\mathscr{N} \sim$ 2 in both limit cases. This symmetry is due to similar volume fractions of gas and solid in WP ( $\varepsilon=0.60)$, as opposed to their disproportion in $\mathrm{BP}(\varepsilon=0.28)$.

(d) Solid is predominant in BP. Therefore, when solid is the most conducting phase, tortuosity is minimal and $\bar{\Lambda}$ is not much smaller than $\langle\lambda\rangle$. It is even very close to it in the most favorable direction with $\Lambda_{\text {Max }} /\langle\lambda\rangle \approx 0.95$, and still nearly half of it in the least favorable one. Conversely, $\Lambda_{\operatorname{Max}}$, $\bar{\Lambda}$ and $\Lambda_{\text {Min }}$ are of the order of $\langle\lambda\rangle$ divided by 10 , 20 and 200, respectively, when gas is the most conducting phase. (e) Even though $\varepsilon_{g}$ is larger than $\varepsilon_{s}$ in WP, the asymptote of the normalized conductivity is smaller for $\lambda_{g} \gg \lambda_{s}$ than that for $\lambda_{s} \gg \lambda_{g}$, for both the mean, maximal and minimal values. This points at a better connectivity of the solid phase, despite its lesser fraction, which results from the morphology: WP resemble gaseous, poorly connected inclusions in a continuous, well connected solid. This morphological feature also applies to BP and contributes, on top of its smaller $\varepsilon_{g}$, to the strong disymmetry of the curves in Fig.3.

Figure 5 for the rock samples FS and BS displays only $\bar{\Lambda}$ because their anisotropy is small $(\mathscr{N} \sim$ at most 1.2 , when $\lambda_{s} \ll \lambda_{g}$ ) and the plots of $\Lambda_{\text {Max }}$ and $\Lambda_{\text {Min }}$ are very similar. In both cases, solid is predominant $\left(\varepsilon_{s}=0.93\right.$ and 0.77 ), and $\bar{\Lambda}$ is of the order of $\langle\lambda\rangle$ when it is the most conducting phase. In the opposite case of $\lambda_{g} \gg \lambda_{s}, \bar{\Lambda}$ is about $1 / 20$ (FS) or $1 / 3$ (BS) $\langle\lambda\rangle$, which is similar to BP and WP, even though their porosities are much smaller. This is because the pores, which are the intergranular space in a partially cemented sand bed, are better connected than the inclusions in the degraded polymers.

The volume fractions of each phase and the connectivity issues due to the pore morphology mentioned in $(d, e)$ are expected to influence the impact of the BC's on the determination of $\boldsymbol{\Lambda}$. When the most conducting phase is predominant and well connected, artefacts such as those illustrated in Fig.2(a,b) are expected to be minimized. Conversely, strong effects are probable when a poorly connected phase with low volume fraction is the most conducting. The comparison of the data in Figs.3-5 confirm these expectations. The relative deviations between the predictions from various BC's are small when $\lambda_{s} \gg \lambda_{g}$ and very large when $\lambda_{g} \gg \lambda_{s}$ in BP (Fig.3). They are also quite significant in FS and BS when $\lambda_{g} \gg \lambda_{s}$ (Fig.5), and in both limit cases in WP (Fig.4), and slightly more so when $\lambda_{g} \gg \lambda_{s}$. It is also observed that as a rule the predictions of $[\mathrm{P} / \mathrm{P}]$ and $[\mathrm{E}+\mathrm{P}]$ are in good agreement, that those of $[\mathrm{D} / \mathrm{P}]$ somewhat deviates from them, and that [D/D] can yield very different and always larger results. This is a consequence of the artefact discussed in Sec.II F and illustrated in Fig.2d. The apparent conducting skin along the transverse boundaries provides an additional path for the heat flux.

Of course, comparing the eigenvalues of $\Lambda$ is meaningful only if the associated eigendirections are similar. This has been checked, and whereas the eigendirections of the tensors resulting from different BC's are not exactly identical, their deviations are small. For instance, in the most severe case of BP with $\lambda_{s} / \lambda_{g}=10^{-4}$ where very large discrepancies between the conductivity predictions for the various BC's are observed in Fig.3, the directions associated with $\Lambda_{M i n}$ for $[\mathrm{P} / \mathrm{P}]$, $[\mathrm{D} / \mathrm{P}]$ and $[\mathrm{E}+\mathrm{P}]$ never deviate by more than $2^{\circ}$, and that for [D/D] is always within 3 to $4^{\circ}$ from the others. Similarly, in sample WP with $\lambda_{s} / \lambda_{g}=10^{-4}$, the four directions of $\Lambda_{M i n}$ are within less than $3^{\circ}$ from eachother. 


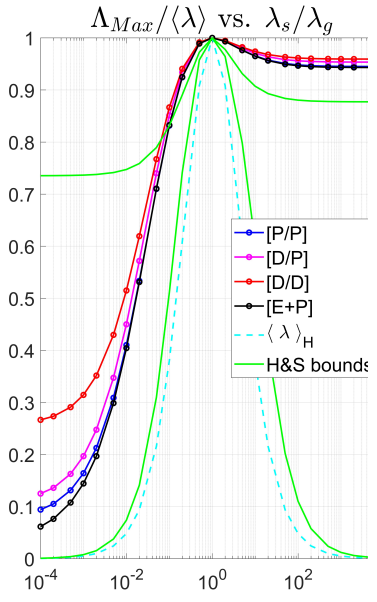

(a)

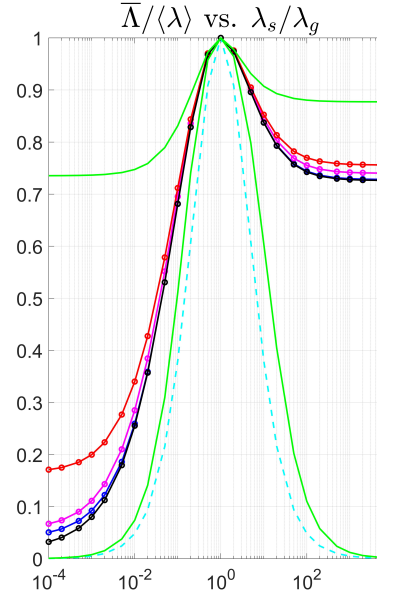

(b)

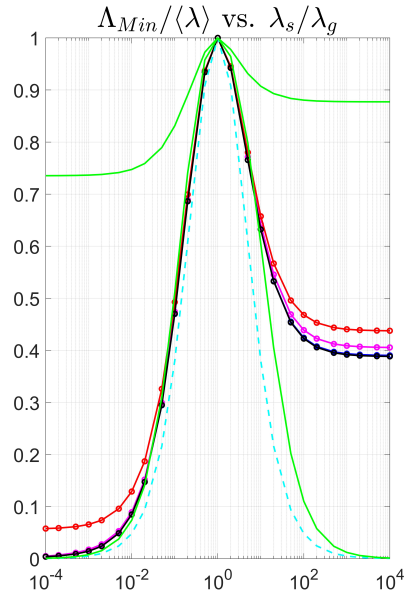

(c)

FIG. 3. Largest eigenvalue $\Lambda_{\text {Max }}$ (a), mean diagonal term $\bar{\Lambda}$ (b) and smallest eigenvalue $\Lambda_{\text {Min }}$ of $\Lambda$, normalized by the volume average $\langle\lambda\rangle$, obtained with various BC's in sample BP, as functions of $\lambda_{s} / \lambda_{g}$. Hashin \& Shtrikman's bounds and the harmonic average $\langle\lambda\rangle_{H}$ are shown for comparison.

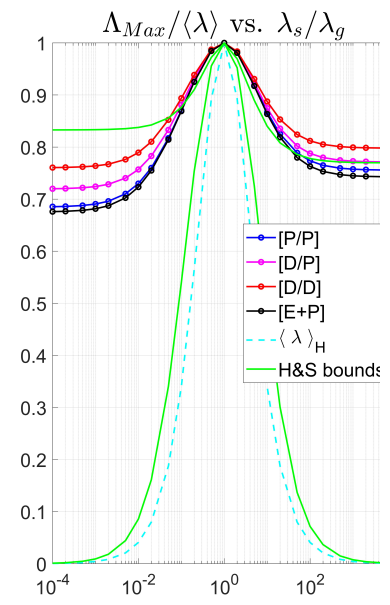

(a)

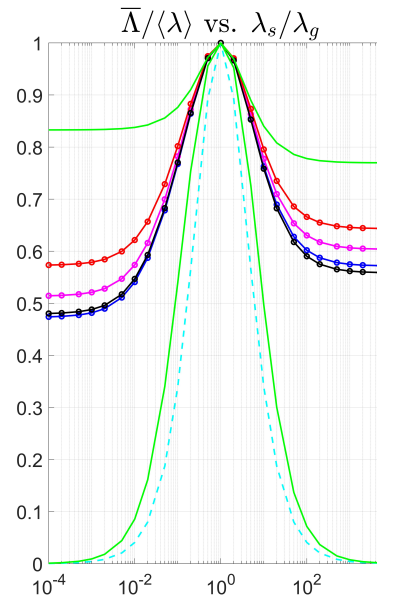

(b)

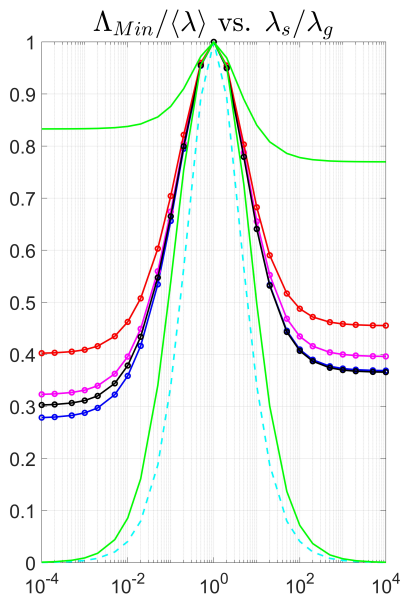

(c)

FIG. 4. Largest eigenvalue $\Lambda_{\operatorname{Max}}$ (a), mean diagonal term $\bar{\Lambda}$ (b) and smallest eigenvalue $\Lambda_{\text {Min }}$ of $\boldsymbol{\Lambda}$, normalized by the volume average $\langle\lambda\rangle$, obtained with various BC's in sample WP, as functions of $\lambda_{s} / \lambda_{g}$. Hashin \& Shtrikman's bounds and the harmonic average $\langle\lambda\rangle_{H}$ are shown for comparison.

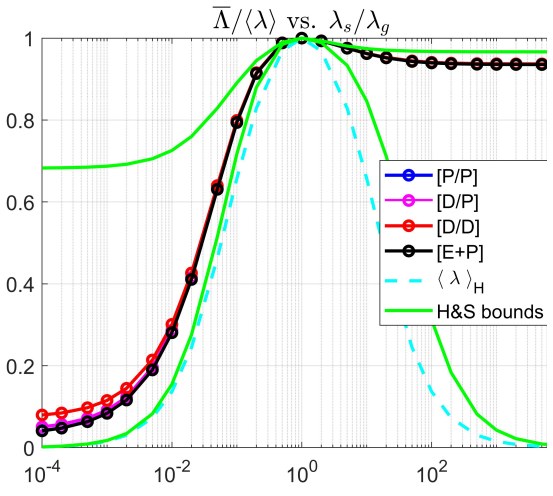

(a: FS)

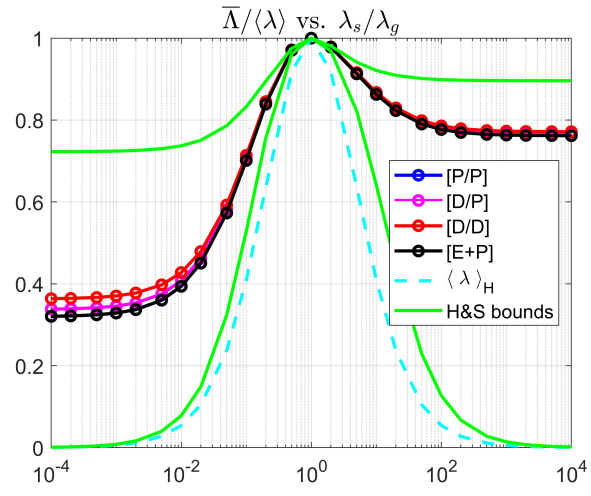

(b: BS)

FIG. 5. Mean diagonal term $\bar{\Lambda}$ normalized by the volume average $\langle\lambda\rangle$, obtained with various BC's in samples FS (a) and BS (b), as functions of $\lambda_{s} / \lambda_{g}$. Hashin \& Shtrikman's bounds and $\langle\lambda\rangle_{H}$ are shown for comparison. 


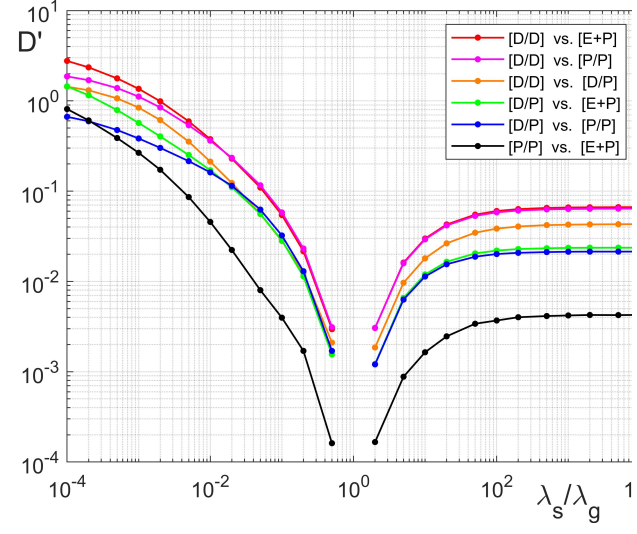

(a: BP)

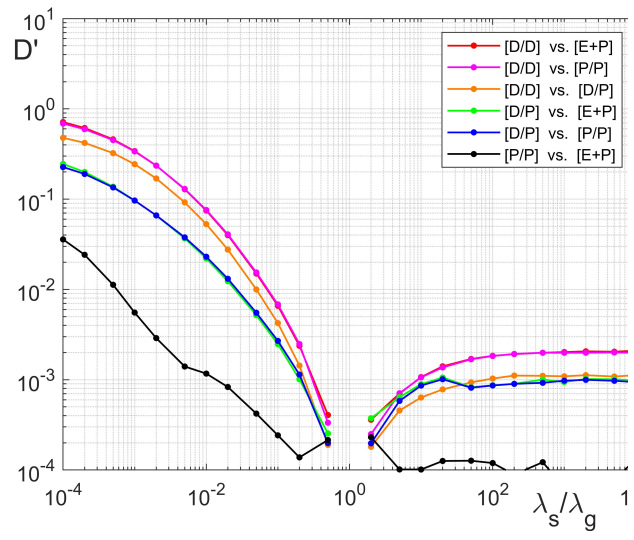

(c: FS)

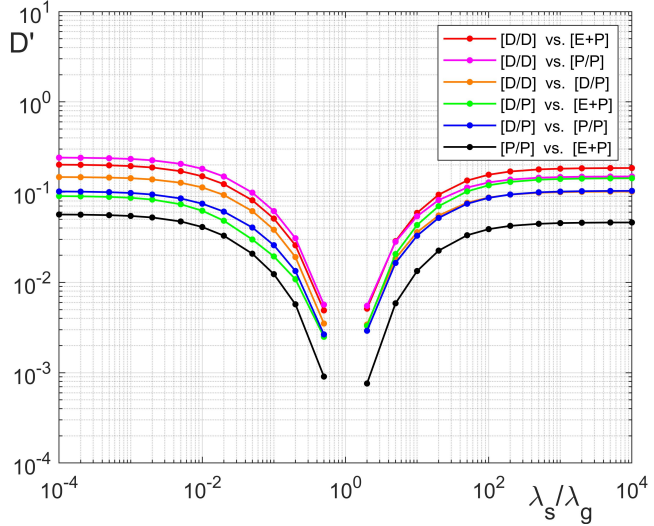

(b: WP)

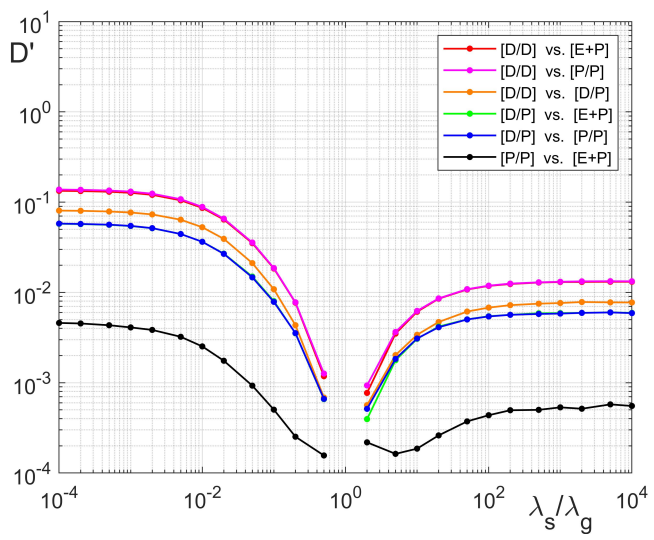

(d: BS)

FIG. 6. Distance $\mathscr{D}^{\prime}\left(\boldsymbol{\Lambda}_{1}, \boldsymbol{\Lambda}_{2}\right)$ between the conductivity tensors obtained in the four samples from tomographic images with various pairs of BC's, as functions of the conductivity contrast ratio $\lambda_{s} / \lambda_{g}$.

Anyway, the relative difference between two tensors quantified by $\mathscr{D}^{\prime}$ defined in (13) incorporates the differences between their eigenvalues but also the deviations between their eigenvectors. It is used in the following to analyze quantatively the features mentioned qualitatively in the above. The distance $\mathscr{D}^{\prime}\left(\boldsymbol{\Lambda}_{1}, \boldsymbol{\Lambda}_{2}\right)$ between the conductivity tensors obtained in the four samples from tomographic images BP, WP, FS and BS, with the $\boldsymbol{\Lambda}_{i}$ 's resulting from calculations with four kinds of BC's, are plotted in Fig.6 as functions of $\lambda_{s} / \lambda_{g}$.

Unsurprisingly, the distance $\mathscr{D}^{\prime}$ between the predictions increases with the contrast in the conductivities of the components, and reaches asymptotes when the contrast is large. This is observed in all cases except for BP and FS where the asymptote for $\lambda_{s} / \lambda_{g} \rightarrow 0$ is not reached yet in the investigated range. When one of the phases is present in a large amount (such as the solid with $\varepsilon_{s}=0.72,0.93$ or 0.77 in BP, FS and BS) and is also the most conducting component, no dramatic influence of the BC's is to be feared. $\mathscr{D}^{\prime}$ never exceeds a few percents in these cases. Conversely, when the most conducting phase is present in a small amount (FS, with $\varepsilon_{g}=0.07$ ) or in a moderate amount but poorly connected (BP, with $\left.\varepsilon_{g}=0.28\right)$ a very strong influence with differences $\mathscr{D}^{\prime}$ of order $\mathrm{O}(1)$ is observed. WP, where both phases are present in important amounts
( $\left.\varepsilon_{g}=0.60, \varepsilon_{s}=0.40\right)$ is an intermediate case, as well as $\mathrm{BS}$ when the gas is very conducting ( $\varepsilon_{g}=0.23$ but well connected). In these case, significant differences occur but do not exceed $\approx 20 \%$.

These observations provide a clue for a practical, $a$ priori detection of cases where caution is required. The at-risk situations are associated with a scarce and/or poorly connected conducting phase. They can be detected by an effective conductivity much smaller than the volume average $\langle\lambda\rangle$, especially regarding its smallest eigenvalue $\Lambda_{\text {Min }}$. All the data for $\mathscr{D}^{\prime}\left(\boldsymbol{\Lambda}_{1}, \boldsymbol{\Lambda}_{2}\right)$ from Figs.3 and 5, for all pairs of BC's and ratios $\lambda_{s} / \lambda_{g}$ in samples BP, FS and BS are plotted in Fig.7 as functions of $\Lambda_{\text {Min }} /\langle\lambda\rangle$ (the smallest of $\Lambda_{M i n, 1}$ and $\Lambda_{\operatorname{Min}, 2}$, but it makes a difference only when is very large). Sample WP is not included because it is too small to provided reliable predictions, regardless of the choice BC (see fig.1c,d).

The results show the same hierarchy as in Fig.6, with the largest $\mathscr{D}^{\prime}$ for pairs of BC's involving [D/D], but when $\Lambda_{\text {Min }} /\langle\lambda\rangle$ is small, $\mathscr{D}^{\prime}$ scales roughly as $\langle\lambda\rangle / \Lambda_{\text {Min }}$ in all cases. A "safe zone" exist for $\Lambda_{\text {Min }} /\langle\lambda\rangle \geq 1 / 2$, where $\mathscr{D}^{\prime}$ is always smaller than 0.05 . Hence, if a calculation with some condition $\mathrm{BC}_{1}$ yields $\Lambda_{1}$, it can be confidently assumed that the fluxes predicted from $\Lambda_{1}$ for any gradient direction never de- 


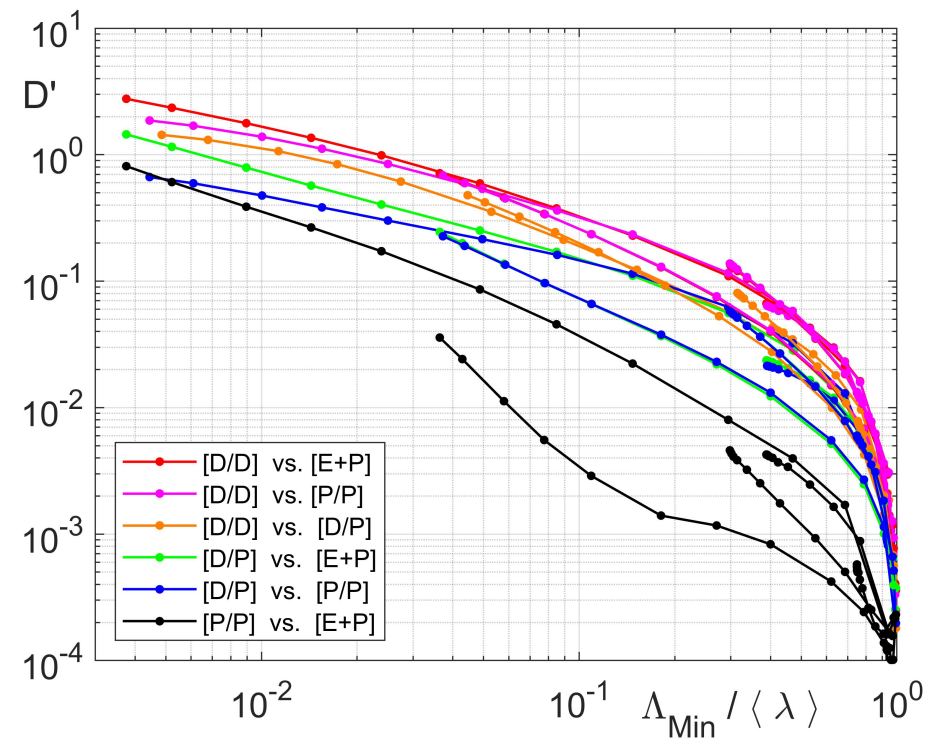

FIG. 7. Distance $\mathscr{D}^{\prime}\left(\boldsymbol{\Lambda}_{1}, \boldsymbol{\Lambda}_{2}\right)$ as a function of $\Lambda_{M i n} /\langle\lambda\rangle$. The results for all pairs of BC's and all ratios $\lambda_{s} / \lambda_{g}$ in BP, FS and BS are included. The lines join data for all ratios $\lambda_{s} / \lambda_{g}$ in a particular sample and pair of BC's.

viate by more that $5 \%$ from those of a tensor resulting from a different upscaling protocol with any other BC. Conversely, caution is required if it is found that $\Lambda_{\text {Min }, 1} /\langle\lambda\rangle<1 / 2$, since another protocol might yield a significantly different $\Lambda$. As a rule of thumb, its deviation $\mathscr{D}^{\prime}$ from $\Lambda_{1}$ can reach up to $\approx 0.04\langle\lambda\rangle / \Lambda_{\text {Min }}$. Then, depending on the desired degree of confidence, it can be wise to investigate more precisely the impact of the upscaling protocol in the targeted situation.

Note that it is advocated later that using the [D/D] immersion condition is not recommended, because it introduces by itself strong artefacts. It induces thereby large values of $\mathscr{D}^{\prime}$ from the predictions with other BC's, but mostly due to its own identified sources of error. If the pairs of $\mathrm{BC}$ 's involving [D/D] are disregarded in Fig.7, the "safe zone" is somewhat wider, with $\mathscr{D}^{\prime} \leq 0.05$ whenever $\Lambda_{\text {Min }} /\langle\lambda\rangle \geq 1 / 3$. Furthermore, the uncertainty outside this zone is also reduced, with a maximal value of $\mathscr{D}^{\prime}\left(\boldsymbol{\Lambda}, \boldsymbol{\Lambda}_{1}\right)$ about $0.02\langle\lambda\rangle / \Lambda_{\text {Min }}$.

\section{COMPARISON OF LOCAL FIELDS}

Whatever the BC's, $\boldsymbol{\Lambda}_{[B C]}$ is deduced via (10) from the fluxes $\langle\mathbf{q}\rangle$ obtained when imposing a unit mean gradient $\langle\nabla T\rangle$. Thus, comparing $\boldsymbol{\Lambda}_{\left[B C_{1}\right]}$ and $\boldsymbol{\Lambda}_{\left[B C_{2}\right]}$ means comparing the fluxes obtained for an identical $\langle\nabla T\rangle$ with different BC's. From a reversed point of view, the temperature fields corresponding to different BC's but identical mean fluxes can be compared. They are deduced from our data simply by renormalizing the fields so that the mean flux becomes one, i.e., basically by dividing the fields obtained for a unit gradient by the corresponding diagonal component of $\boldsymbol{\Lambda}$. Let $T_{[B C], j}^{(1)}$ denote the field with mean gradient along direction $j$ which yields a unit flux $q_{j}$ under the boundary con- ditions [BC]. Two such fields for different BC's can then be compared, and their difference $\widetilde{\delta T}_{\left[B C_{1}\right],\left[B C_{2}\right], j}$ is made dimensionless by dividing it by the overall drop $\Delta T^{(1)}$ over the whole sample.

Examples are displayed in Fig.8 for the cross-section of BS shown in (a), when $\lambda_{s} / \lambda_{g}=10^{-2}$. Large deviations between the fields for $[\mathrm{P} / \mathrm{P}]$ and $[\mathrm{D} / \mathrm{P}]$ are observed in (b) near the inlet and outlet faces (exceeding $5 \%$ of $\Delta T^{(1)}$ ), as expected since this is where the BC's differ (periodicity or Dirichlet). Differences also exist along the transverse boundaries, even though the boundary conditions are identical. This is because the global differences in flow pattern (including a marginal deviation of the overall mean flux, in this slightly anisotropic sample) induce slightly different fluxes across them. Since they often have to cross constrictions due to mismatches of the aperiodic geometry, this translates into noticeable differences in the local gradients. However, the deviations near the transverse boundaries are much weaker and reach a much smaller depth than those near the inlet and outlet boundaries. The comparison in (c) of $[\mathrm{P} / \mathrm{P}]$ and $[\mathrm{E}+\mathrm{P}]$ yields a similar picture, but with deviations of a smaller amplitude. In all comparisons involving $[\mathrm{P} / \mathrm{P}],[\mathrm{D} / \mathrm{P}]$ and $[\mathrm{E}+\mathrm{P}]$, the fields are nearly identical in a very large central part of the domain.

However, the comparison of $[\mathrm{D} / \mathrm{D}]$ with $[\mathrm{D} / \mathrm{P}]$ in Fig.8d (as well as the confrontations of $[D / D]$ with $[\mathrm{P} / \mathrm{P}]$ and $[\mathrm{E}+\mathrm{P}]$, not shown) is quite different. As noted in Sec.III, the immersion conditions increase the effective conductivity due to a supplementary conduction in a skin along the transverse boundaries. Therefore, the overall mean flux $\left\langle q_{j}\right\rangle$ is not representative of the flux in the bulk of the sample, and consequently, after calibration of the T-field into $T_{[D / D], j}^{(1)}$, the mean gradient in the bulk does not correspond to a unit mean flux. As a result, the good agreements of the fields in the cen- 


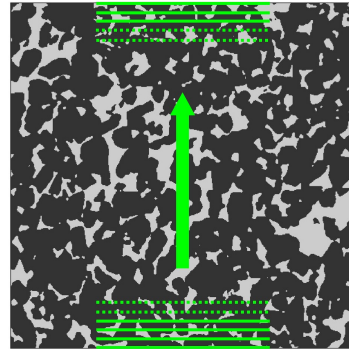

(a)

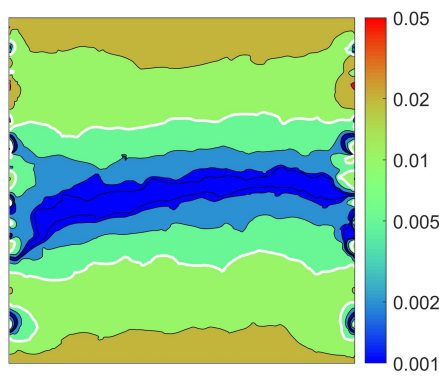

(d)

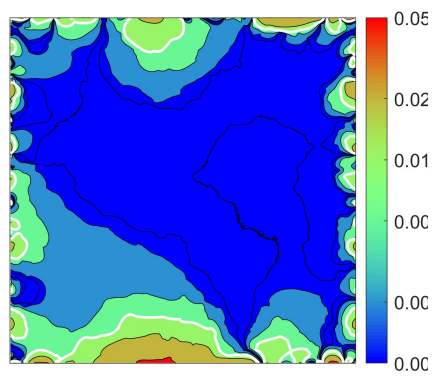

(b)

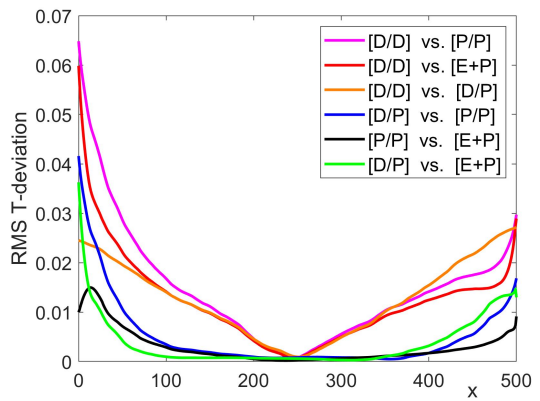

(e)

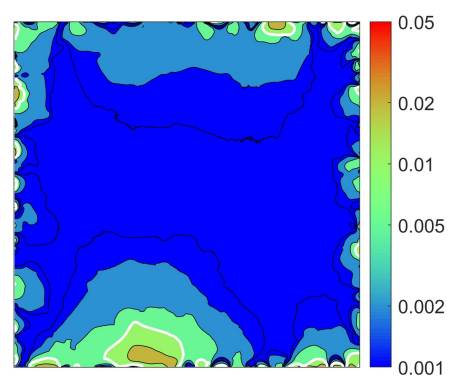

(c)

FIG. 8. Comparaison of local fields. Exemple for the cross-section (a) through sample BS. The mean gradient is vertical (arrow) and $\lambda_{s} / \lambda_{g}=10^{-2}$. Maps of $|\widetilde{\delta T}|$ for BC's $[\mathrm{P} / \mathrm{P}]$ and $[\mathrm{D} / \mathrm{P}](\mathrm{b}),[\mathrm{P} / \mathrm{P}]$ and $[\mathrm{E}+\mathrm{P}](\mathrm{c})$ and $[\mathrm{D} / \mathrm{P}]$ and $[\mathrm{D} / \mathrm{D}](\mathrm{d})$, in logscale. The white lines corresponds to $10^{-2}$. Profiles of the RMS mean $\langle\widetilde{\delta T}\rangle_{2}$ along the gradient direction (e). The transverse averages are taken in the central half of the domain, i.e., over the green streaks in (a).

tral regions of Figs.8b,c is replaced by the difference of two different linear trends in Fig.8d.

This is made more explicit in Fig.8e. The transverse RMS average $\langle\widetilde{\delta T}\rangle_{2}$, measured over transverse segments in the middle half of the sample (green streaks in Fig.8a), is plotted as a function of the position in the direction parallel to the flow. For the comparisons of $[\mathrm{P} / \mathrm{P}],[\mathrm{D} / \mathrm{P}]$ and $[\mathrm{E}+\mathrm{P}]$, large mean deviations take place at the inlet and oulet, which rapidly decrease in the depth of the sample, and $\langle\widetilde{\delta T}\rangle_{2}$ nearly vanishes in a broad central region. For the comparisons of [D/D] with other BC's, the same pattern is superimposed with a linear function of position. Note that this could be cured by renormalizing the fields for [D/D] by a flux averaged over a region excluding the vicinity of the transverse boundaries instead of the overall $\langle q\rangle$ when constructing $T_{[D / D], j}^{(1)}$. This would result in a good match with other BC's in the central region and large deviations $\widetilde{\delta T}$ along the sides. This is the basic idea of the treatments in Sec.V but it was not implemented for the local comparisons which are discussed here.

The treatments of Fig.8 were repeated for samples $\mathrm{BP}, \mathrm{WP}, \mathrm{FS}$ and BS, all values of the contrast ratio $\lambda_{s} / \lambda_{g}$, mean gradients along $x, y$ and $z$, and all BC's. In each case and for each pair of BC's, $\widetilde{\delta T}$ was measured in six sections parallel to the mean gradient (e.g., planes $y / L_{y}$ and $z / L_{z}=1 / 4,1 / 2$ and $3 / 4$ when $\langle\nabla T\rangle \| x)$. The data from the six sections, folded to make use of the fore/aft symmetry, are included in the
RMS average $\langle\widetilde{\delta T}\rangle_{2}$.

The results are shown for sample BS in Fig9. Since BS is nearly isotropic, the data for $\langle\nabla T\rangle \| x, y$ and $z$ have also been merged when evaluating $\langle\widetilde{\delta T}\rangle_{2}$. Small fluctuations (never larger than $\approx 10^{-3}$ ) are sometimes visible when $\lambda_{s} / \lambda_{g} \sim 10^{4}$. These local instabilities in the solver are caused by a very poor conditionning of the problem but do not induce any macroscopic effect. The general picture is identical to that for one section of BP in Fig.8e, with a much reduced noise due to the larger sampling domain (9 sections vs. a single one, and BS is also larger than BP in terms of $L / l_{c}$, see Table I). The same features are obtained, with significant deviations at the inlet and outlet, rapidly decreasing towards a nearly vanishing value in a wide central region as long as the pair of BC's does not involve [D/D]. If it does, a linear function of position is again superimposed with the former pattern. In the comparison of $[D / D]$ vs. $[D / P]$, this linear part induced by the lateral conducting skin introduced by $[\mathrm{D} / \mathrm{D}]$ is actually the only contribution to $\langle\widetilde{\delta T}\rangle_{2}$, since identical Dirichlet conditions are imposed at the upstream and downstream faces. Unsurprisingly, the deviations increase with the conductivity contrast, and reach a higher level when conduction relies on the phase with the smallest volume fraction (the gas, in $\mathrm{BS}$, with $\varepsilon_{g}=0.23$ ) than in the opposite case.

To conclude with the comparisons of local fields, the data for FS, BS and BP are summarized in Fig.10. Attention is focused on the region near the inlet/oulet 

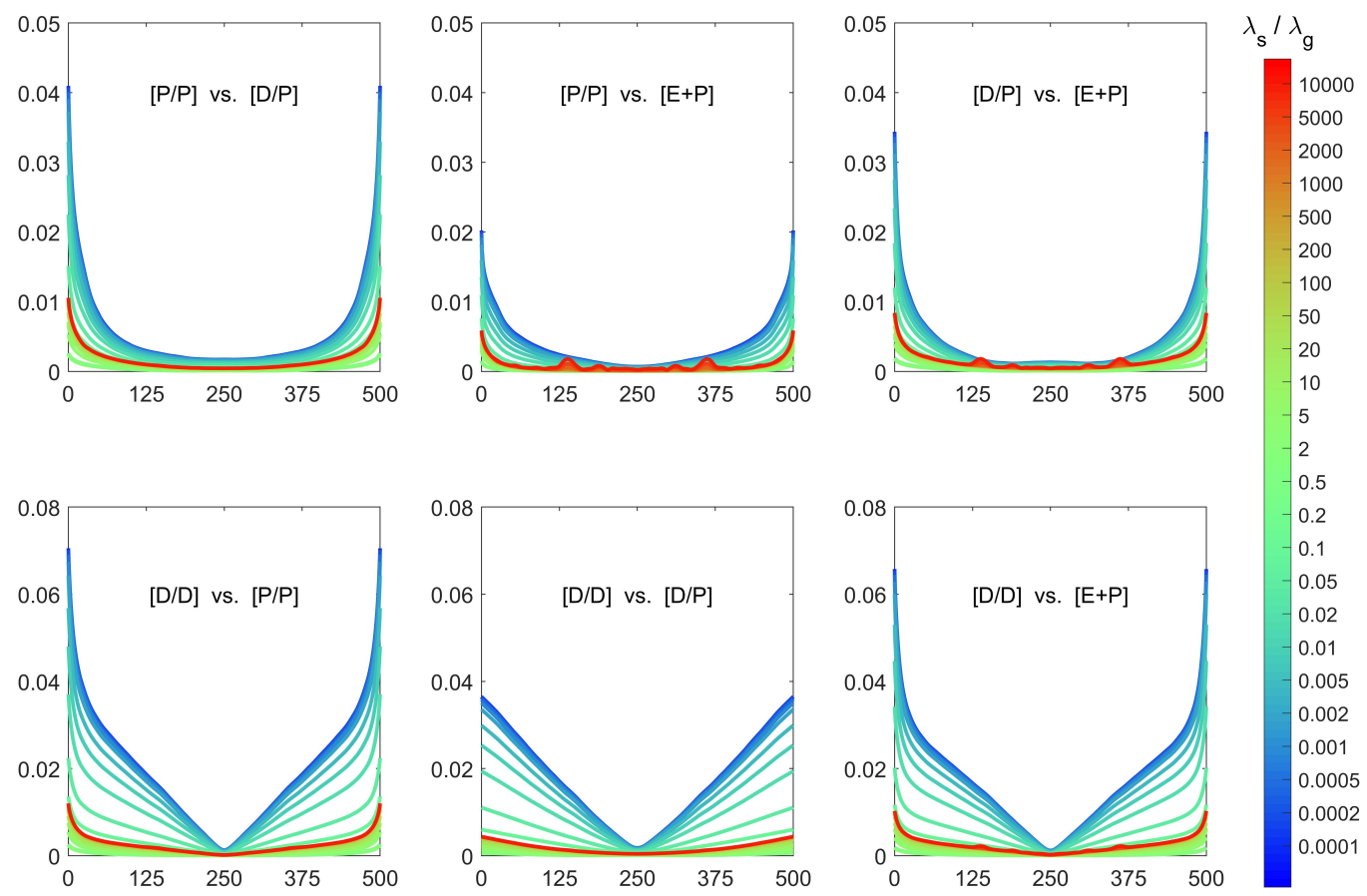

FIG. 9. Profiles of $\langle\widetilde{\delta T}\rangle_{2}$ along the mean gradient direction in BS, for pairs of BC's involving (bottom row) or not (top row) [D/D], and conductivity ratios $\lambda_{s} / \lambda_{g}$ indicated by the color code.

faces, and $\langle\widetilde{\delta T}\rangle_{2}$ is plotted as a function of depth $d$ for pairs of $\mathrm{BC}$ 's involving $[\mathrm{P} / \mathrm{P}],[\mathrm{D} / \mathrm{P}]$ and $[\mathrm{E}+\mathrm{P}]$. The immersion conditions [D/D] are not included since the fields cannot be calibrated for a meaninful comparison. The data for $\langle\nabla T\rangle \| x, y$ and $z$ are averaged for FS and BS. However, the data for $\langle\nabla T\rangle \| x$ or $y$ and those for $\langle\nabla T\rangle \| z$ (respectively parallel and normal to the plane of the oblate bubbles visible in Fig.1) are treated separately in the anisotropic sample $\mathrm{BP}$.

An exponential decay establishes rapidly in FS with a decay length $l_{d}$ remarkably independent of the BC's and $\lambda_{s} / \lambda_{g}$,

$$
\langle\widetilde{\delta T}\rangle_{2} \propto \mathrm{e}^{-d / l_{d}} \quad \text { for } d \gtrsim 4 l_{c}, \text { with } l_{d} \approx 19 l_{c} \text { in FS }
$$

The corresponding slope is indicated by broken lines in Fig.10. Slight deviations from this general trend take place very near to the sample faces. A plot as a function of $\lambda_{s} / \lambda_{g}$ (not shown) of $\langle\widetilde{\delta T}\rangle_{2}$ at the origin $d=0$, i.e., of the RMS $T$-deviations measured at the inlet/outlet faces, looks very similar to that of the tensor distance $\mathscr{D}^{\prime}$ in Fig.6 (this remark applies to all the samples considered here). Similar comments apply to BS. The exponential regime establishes at a depth of the order of $5 l_{c}$, with a decay length $l_{d} \approx 13 l_{c}$. Thus, it seems that two scales are involved in the screening of the BC's influence within the medium. One might be related to the texture, i.e., to the typical size of the pores and of the grains underlying the structure of these sandstones, governing the impact of the geometrical mismatches at the sample boundaries and the range of the strong local disturbances that they induce. Then, these disturbances are damped on a larger scale, associated with the orga- nization of the more or less conducting regions, which might span several grain sizes.

The case of sample BP is more complicated for several reasons. First, it is smaller in terms of statistical content, i.e., of its relative size $L / l_{c}$, which favors strong fluctuations. Then, unlike FS and BS, sample BP contains multiscale features (bubbles). The integral correlation lengths given in Table I are associated with the larger ones. Finally, BP is anisotropic, which is why the data for $\langle\nabla T\rangle$ in the plane of the large bubbles or normal to it are analyzed separately (last two rows in Fig.10). Nevertheless, the long range decay of $\langle\widetilde{\delta T}\rangle_{2}$ is well represented by the exponential form of (15), with a same value of $l_{d} \approx 3 l_{c x} \approx 13 l_{c z}$ in most cases. Exceptions result from statistical fluctuations in some cases. These fluctuations make also difficult to quantify precisely where the exponential regime establishes. However, whereas $l_{d}$ is fairly constant whenever $\lambda_{s} / \lambda_{g} \geq 10^{-2}$, it tends to significantly increase in most comparisons when the solid becomes insulating, with $\lambda_{s} / \lambda_{g} \lesssim 10^{-3}$. For instance, it reaches $l_{d} \approx 7 l_{c x} \approx 31$ $l_{c z}$ in the comparison of [P/P] vs. [D/P] for $\langle\nabla T\rangle \perp z$ (dotted line in Fig.10). The reason for this transition is not elucidated.

\section{UNDERSAMPLING : MEASUREMENTS IN INNER SUBDOMAINS}

The conductivity tensors associated with central subblocks of the samples have been systematically evaluated according to the procedure described in Section II H. During the calculations for each individual case, 

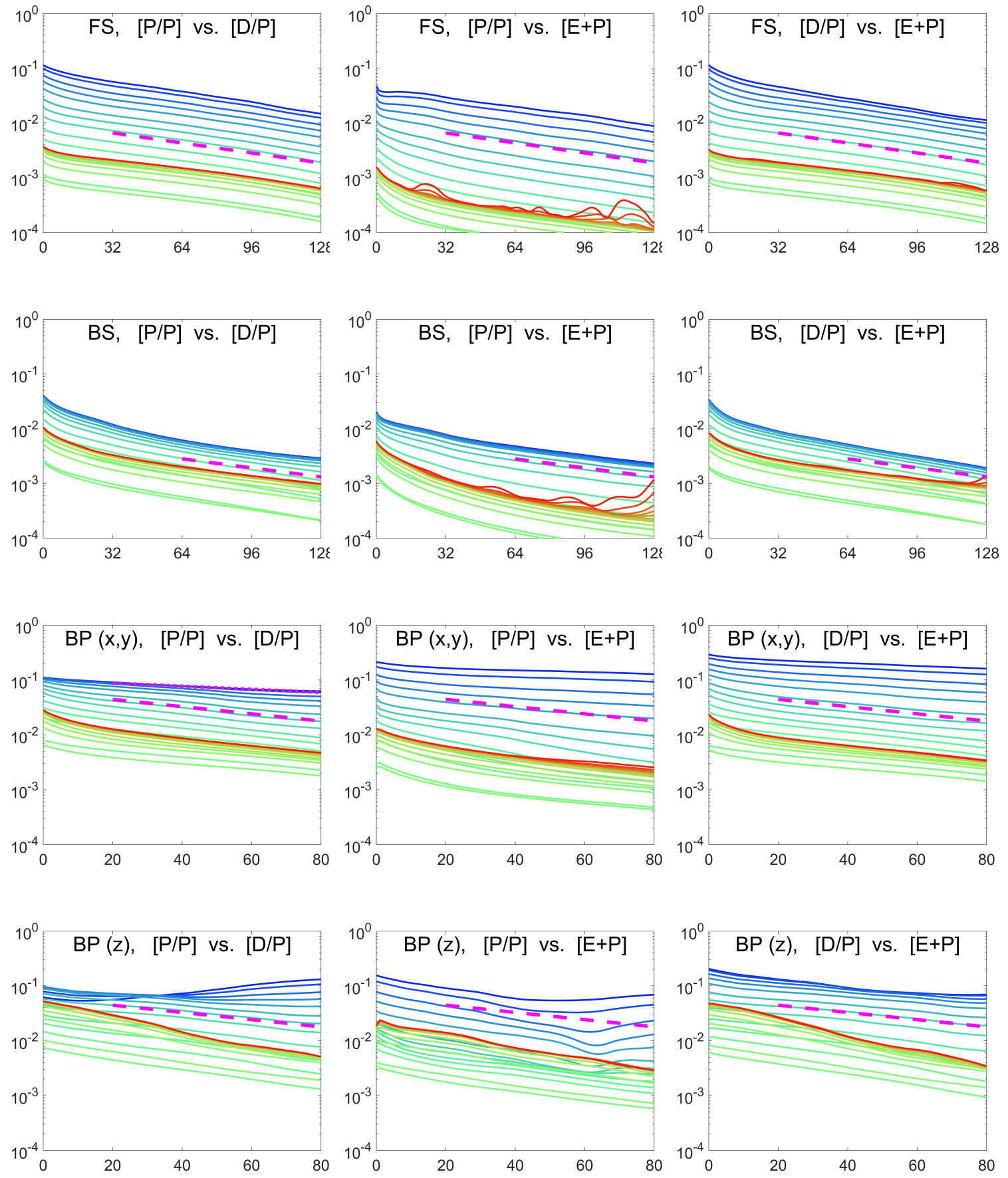

FIG. 10. $\langle\widetilde{\delta T}\rangle_{2}$ as a function of depth $d$ measured from the inlet/outlet faces, for various pairs of BC's (columns), in samples FS (top row), BS (second row), and BP (bottom rows). Data for $\langle\nabla T\rangle$ normal and parallel to the $z$-axis are presented separately for BP. Colors correspond to the ratio $\lambda_{s} / \lambda_{g}$, with the same convention as in Fig.9. Broken lines indicate the slopes corresponding to (15) with $l_{d}=19 l_{c}(\mathrm{FS}), 13 l_{c}$ (BS) or $13 l_{c z}(\mathrm{BP})$. The dotted line for $\mathrm{BP}(x, y)$ corresponds to $l_{d}=31 l_{c z}$.

the mean flux $\langle\mathbf{q}\rangle_{c}$ and gradient $\langle\nabla T\rangle_{c}$ have been measured in a series of concentric subdomains $\Omega_{c}(M)$ obtained by removing from $\Omega$ a peripheral layer with thickness $M$. A tensor $\boldsymbol{\Lambda}_{[B C], c}(M)$ can be deduced via (10) from the set of $\langle\mathbf{q}\rangle_{c}$ and $\langle\nabla T\rangle_{c}$ vectors measured in $\Omega_{c}(M)$ when solving problem (1) submitted to boundary conditions $[\mathrm{BC}]$ at $\partial \Omega$ with $\langle\nabla T\rangle$ oriented successively along the $x-, y$ - and $z$-axes.

Results are presented in Fig.11 for the most extreme values of the contrast ratio, with $\lambda_{g}=1$ and $\lambda_{s}=10^{ \pm 4}$, in samples FS, BS and BP. Sample WP is not considered, since it is too small to expect that any sub-block taken from it can be representative. The volume average $\langle\lambda\rangle_{c}$ in $\Omega_{c}$ is considered first (left column in Fig.11), to check if the sub-block content remains representative of the whole sample. Note that $\langle\lambda\rangle_{c}$ is nearly equal to the porosity when $\lambda_{s}=10^{-4}$.

The fluctuations are very small when $M \leq 110 \approx 28$ 

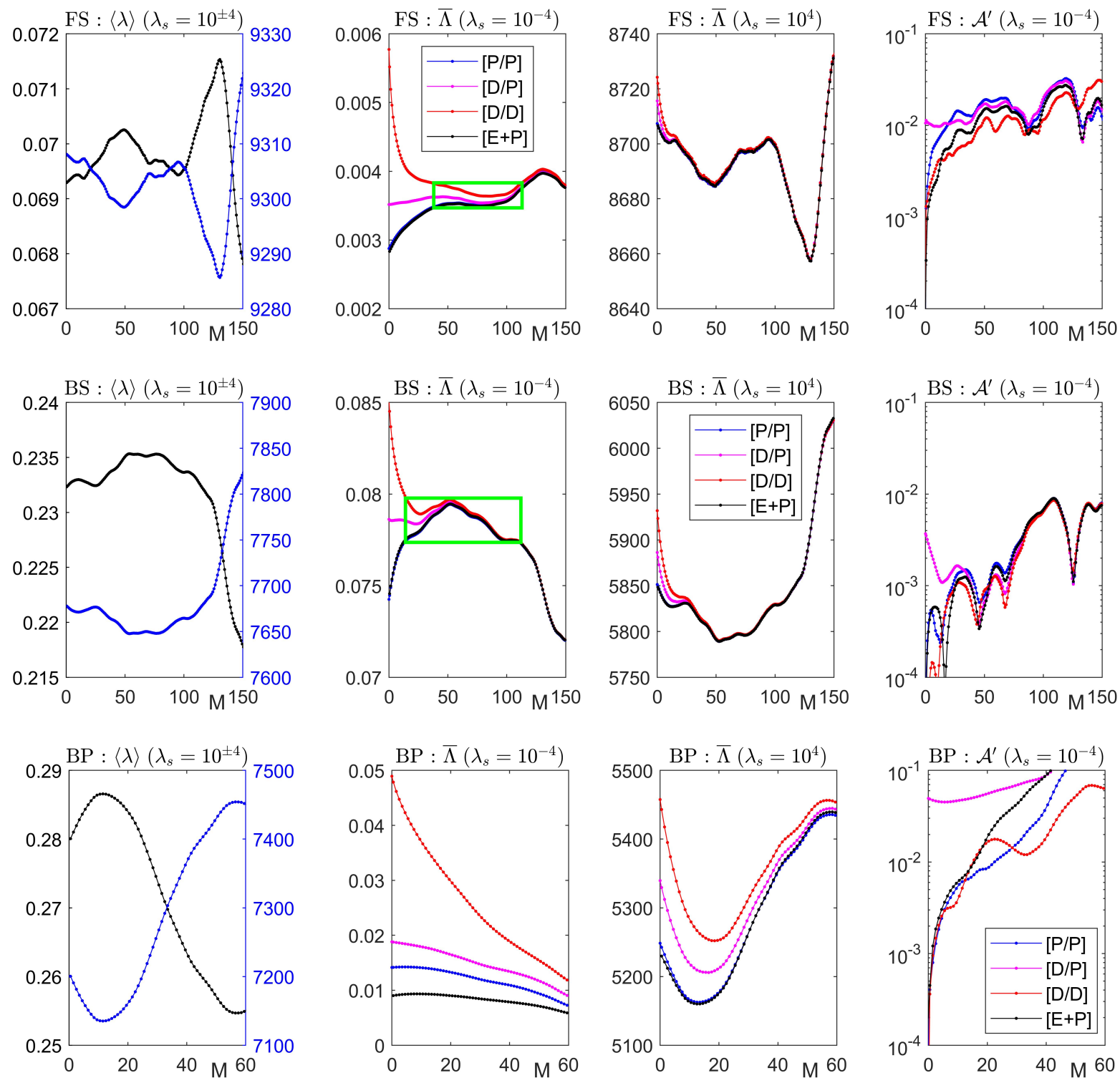

FIG. 11. Measurements in inner domains $\Omega_{c}(M)$, for samples FS (top row), BS (middle row) and BP (bottom row), as functions of the removed layer thickness $M$. The volume averages $\langle\lambda\rangle_{c}$ for $\lambda_{g}=1$ and $\lambda_{s}=10^{ \pm 4}$ (left column). The mean conductivity $\bar{\Lambda}_{c}$ for various BC's, $\lambda_{g}=1$ and $\lambda_{s}=10^{ \pm 4}$ (middle columns). All the values in the green boxes are equal with $\pm 5 \%$ (FS) or $\pm 1.5 \%$ (BS). The asymmetry index $\mathscr{A}_{c}^{\prime}$ for various BC's, $\lambda_{g}=1$ and $\lambda_{s}=10^{4}$ (right column).

$l_{c}$ in FS and $M \leq 130 \approx 20 l_{c}$ in $\mathrm{BS}$, which is more than the decay length $l_{d}$ of the disturbances measured in Section IV. Conversely, fluctuations are much stronger in the relatively smaller sample BP $\left(L / l_{c} \sim O(10)\right.$ vs. $L / l_{c} \sim O\left(10^{2}\right)$ in FS and BS), with porosity deviations reaching 0.005 when $M \approx 30$, although $\Omega_{c}$ is still larger than $\Omega / 2$. Thus a range of $M$ can exist for FS and BS, thick enough to screen the influence of the BC's while $\Omega_{c}$ remains large enough to be representative, but this is very dubious for BP.

The plots of the mean diagonal terms $\bar{\Lambda}_{c}$ resulting from various overall BC's confirm these expectations. Note first that since the solid is always the most present phase, nothing dramatic happens when it is also the most conducting phase $\left(\lambda_{s}=10^{4}\right.$, third column in Fig.11). For $M=0$, the predictions from the various
BC's differ slightly (FS and BS) or moderately (BP), as already seen in Figs. 3 and 5 for $\lambda_{s} / \lambda_{g} \gg 1$, but very fast, they converge and become simply proportional to $\langle\lambda\rangle_{c}$ measured in the same sub-block, with identical (or nearly identical in BP) proportionality coefficients.

The opposite case of $\lambda_{s}=10^{-4}$ (second column in Fig.11) differs by the much wider spread of the values of $\bar{\Lambda}$ for $M=0$ with various BC's. Still, the same trend is observed. As $M$ increases, $\bar{\Lambda}_{c}$ becomes proportional to $\langle\lambda\rangle_{c}$ with identical coefficients. This regime is reached very soon in $\mathrm{BS}$, at $M \approx 20$, and somewhat later in FS, at $M \approx 40$. However, the initial deviations as so strong and felt so deep in BP that convergence is far from reached in the investigated range of $M$. The unnormalized data for $\bar{\Lambda}_{c}$ in Fig.11 incorporate the combined influence of the BC's and of the variations of 
porosity in $\Omega_{c}(M)$. Of course, they strongly differ in $\mathrm{BP}$, but $\bar{\Lambda}_{c}$ is found fairly constant for all BC's and over a broad range of $M$ in FS and BS (green boxes in the figure), which suggest a fairly robust estimate of an intrinsic $\bar{\Lambda}$ for these materials. However, the plots of $\mathscr{A}_{c}^{\prime}$ (right column in Fig.11) show that the tensors $\bar{\Lambda}_{c}$ present some assymetry. For instance, $\mathscr{A}_{c}^{\prime} \approx 0.03$ in FS and 0.01 in BS when $M \sim 100$. Recall that $\bar{\Lambda}_{c}$ differ by at least that much from any acceptable conductivity tensor, as discussed in Section II I. Therefore, this minimal degree of uncertainty should be kept in mind even if concordant predictions of the effective conductivity are obtained from different upscaling procedures. In addition, the differences in mean diagonal $\bar{\Lambda}$ do not incorporate all the differences between tensors and the full distance $\mathscr{D}^{\prime}$ should be considered for a rigorous assessment.

A more complete set of data covering the whole range of contrasts $\lambda_{s} / \lambda_{g}$ is presented in Fig.12, in terms of the distance $\mathscr{D}^{\prime}\left(\boldsymbol{\Lambda}_{\left[B C_{1}\right], c}, \boldsymbol{\Lambda}_{\left[B C_{2}\right], c}\right)$. This accounts more fully of all the differences between tensors, including in their individual eigenvalues and eigendirections, than the mere comparison of the mean diagonal and indeed, $\mathscr{D}^{\prime}$ is seen to reach sometimes 0.13 in the range corresponding to the green box for FS in Fig.11, where $\bar{\Lambda}_{c}$ remains constant within $\pm 5 \%$.

The general features observed in Fig. 6 for the distances between the overall tensors $\Lambda$ also apply to their values $\boldsymbol{\Lambda}_{c}$ in inner sub-domains. $\mathscr{D}^{\prime}$ increases with the contrast in the component conductivities, and tends toward asymptotic values when the contrast is large. When the solid is the most conducting phase, the pictures obtained for all $\lambda_{s} / \lambda_{g} \geq 10$ are very similar, and only those for $\lambda_{s} / \lambda_{g}=100$ are shown. In the opposite case, convergence is also observed in BS with similar pictures whenever $\lambda_{s} / \lambda_{g} \leq 10^{-2}$. Therefore, the comments made about Fig.11 for $\lambda_{s} / \lambda_{g}=10^{-4}$ apply over this whole range. However, the limit for $\lambda_{s} / \lambda_{g} \rightarrow 0$ is not reached yet in FS and BP when $\lambda_{s} / \lambda_{g}=10^{-4}$, as already observed in Fig.6.

It is also clear from Fig.12 that it is easier to find a range of subdomains where the predictions from all the BC's agree within some prescribed tolerance when the contrast is milder. For instance, the tensor predictions for $M \gtrsim 20$ never deviate by more than $1 \%$ in FS and BS, or $20 \%$ in BP when $\lambda_{s} / \lambda_{g} \geq 10^{-2}$, whereas the deviations reach $50 \%$ (FS), $3 \%$ (BS) or exceed unity (BP) when $\lambda_{s} / \lambda_{g}=10^{-4}$. This illustrate the fact that the search for an REV cannot be conducted by considering only the geometry, and that it also depends on the conductivity constrast.

\section{DISCUSSION AND CONCLUSIONS}

The observations made about Fig.7 provide probably the most interesting clues for direct practical applications. Before elaborating about this, let us bring a few complementary elements. Note first that when the conductivity contrast is very large, its precise value makes ultimately no difference and the macroscopic coefficients are in practice equivalent to those for infinite contrast. For instance, if the solid tends to be insulating (similar developments apply in the opposite limit),

$$
\langle\lambda\rangle \rightarrow \lambda_{g} \varepsilon_{g} \quad \text { and } \quad \Lambda_{[B C]} \rightarrow \lambda_{g} \Lambda_{[B C]}^{(1,0)} \quad \text { as } \lambda_{s} / \lambda_{g} \rightarrow 0
$$

where $\Lambda_{[B C]}^{(1,0)}$ is the tensor obtained when $\lambda_{g}=1$ and $\lambda_{S}=0$. This convergence of $\boldsymbol{\Lambda}$ has been observed in
Figs.3-5, although in some cases, the asymptotic value is not reached yet in the investigated range of contrast. As a direct consequence,

$$
\Lambda_{M i n,[B C]} /\langle\lambda\rangle \rightarrow \Lambda_{M i n,[B C]}^{(1,0)} / \varepsilon_{g} \quad \text { and } \quad \mathscr{D}^{\prime}\left(\Lambda_{[B C 1]}, \Lambda_{[B C 2]}\right) \rightarrow \mathscr{D}^{\prime}\left(\Lambda_{[B C 1]}^{(1,0)}, \Lambda_{[B C 2]}^{(1,0)}\right) \quad \text { as } \lambda_{s} / \lambda_{g} \rightarrow 0
$$

Note that the limit for $\mathscr{D}^{\prime}$ is a finite number, except in the particular case when the long-range connections along the transverse sample boundaries introduced by immersion conditions [D/D] make a non percolating medium to appear percolating. The convergence of $\mathscr{D}^{\prime}$ has been observed in Fig.6, although again, the asymptotic value is not reached yet in the investigated range for some cases. This implies that the curves in Fig.7, which correspond to increasing contrasts for each sample and pair of BC's, have to reach a final point on the left side corresponding to the asymptotic values in (16b). Some of the curves in Fig.6 do indeed reach this limit, while others have not reached it yet. However, even though an asymptotic point is known to exist, the arguments in the above tell nothing about the path followed to reach it and the fact that $\mathscr{D}^{\prime}$ is roughly inversely proportional to $\Lambda_{\mathrm{Min}} /\langle\lambda\rangle$ is a new piece of information. Advantage can be taken from it for instance to infer the expected uncertainty for a strong contrast from available knowledge for a milder one. 

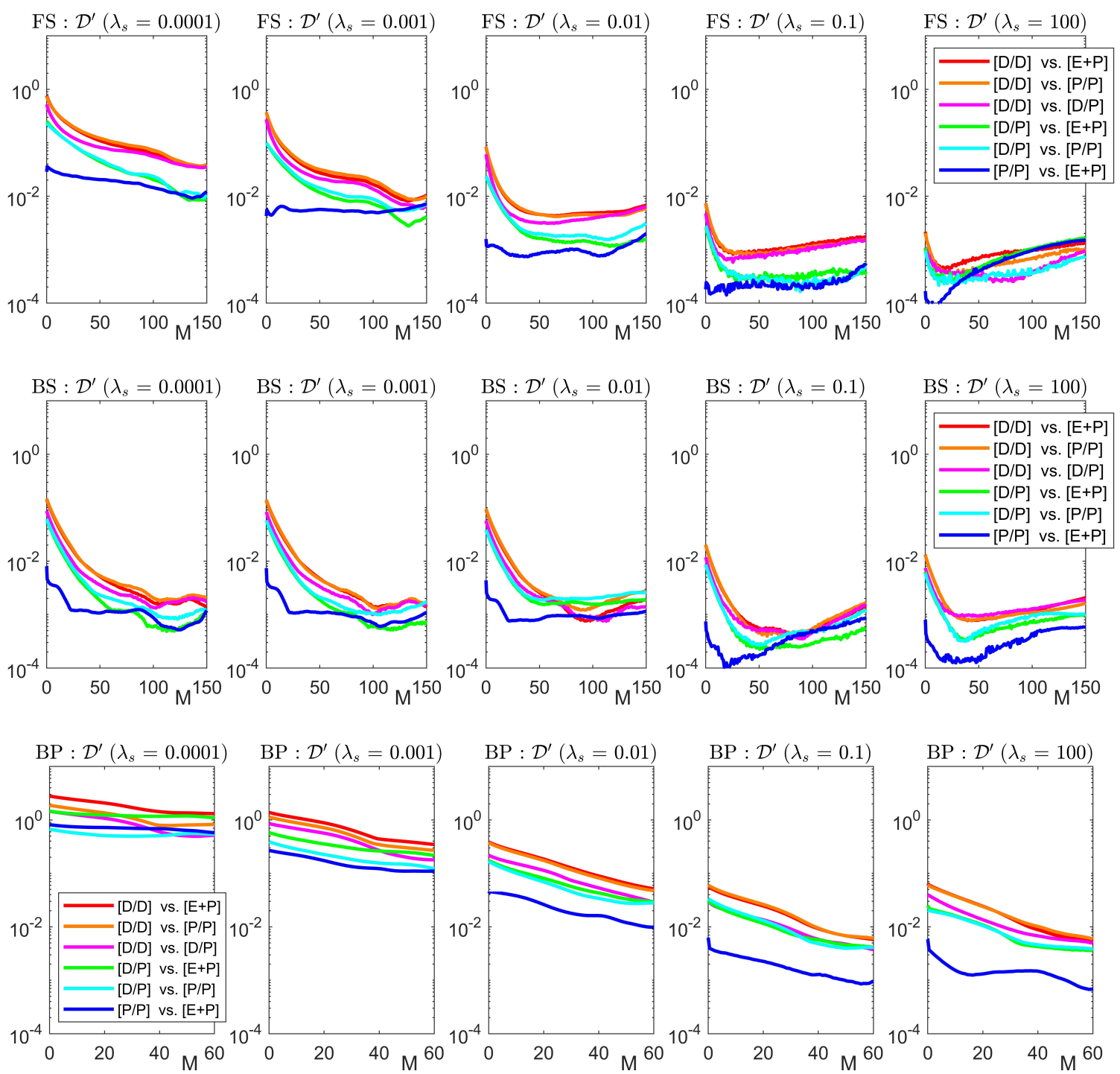

FIG. 12. The distances $\mathscr{D}^{\prime}\left(\boldsymbol{\Lambda}_{\left[B C_{1}\right], c}, \boldsymbol{\Lambda}_{\left[B C_{2}\right], c}\right)$ between the tensors obtained in inner domains $\Omega_{c}(M)$ with various BC's, as functions of the removed layer thickness $M$. Data are for samples FS (top row), BS (middle row) and BP (bottom row), and various ratios $\lambda_{s} / \lambda_{g}$ (columns).

It appears that the representation of $\mathscr{D}^{\prime}$ as a function of $\Lambda_{\mathrm{Min}} /\langle\lambda\rangle$ (as opposed to the plots vs. $\lambda_{s} / \lambda_{g}$ in Fig.6) greatly unifies the data. Note first that each of the curves is actually made of two branches, for $\lambda_{s} / \lambda_{g}$ larger or smaller than 1 , which are very close together. The curves for various samples with the same pair of BC's are also of the same order of magnitude, generally within a factor about 2 (when the deviations are large) or 3 (for smaller deviations).

For any particular sample, the various values of $\mathscr{D}^{\prime}$ at some abscissa in Fig.6 measure the difference of the predictions of $\boldsymbol{\Lambda}$ resulting from various pairs of BC's. Some pairs such as $([\mathrm{P} / \mathrm{P}],[\mathrm{E}+\mathrm{P}])$ are always in a better agreement than others, but the largest $\mathscr{D}^{\prime}$ can be considered as an estimate of the intrinsic uncertainty of the determination of $\boldsymbol{\Lambda}$, since at least two results differ by that much. Thus, only the upper envelop of the curves is to be considered. It was characterized in Section III in a very schematic way. In a "safe region"
$\Lambda_{\text {Min }} /\langle\lambda\rangle \geq 1 / 2, \mathscr{D}^{\prime} \leq 0.05$ for all media and any choice of BC (the choice of the 0.05 threshold is of course arbitrary, different levels of tolerated uncertainty can be chosen depending on the requirements for a specific application). Out of this region, the uncertainty can reach $0.04\langle\lambda\rangle / \Lambda_{\text {Min }}$.

However, what exactly should be contained in the envelop is questionable. It is clear in Fig.7 and in all figures where determinations of $\boldsymbol{\Lambda}$ are compared that the largest $\mathscr{D}^{\prime}$ are always observed when comparing [D/D] to other BC's, and this determines the envelop as quantified in the above. On the other hand, the shortcomings of the immersion conditions have been repeatedly pointed out. By artificial introducing a conducting layer along the transverse sample boundaries, they significantly increase the apparent conductivity. This even prevented in Section IV the comparison of local fields under similar conditions (same overall mean flux). We advocate that the large values of $\mathscr{D}^{\prime}$ associated with 
[D/D] originate in this artefact, that [D/D] should not be used and that there is no need to include them in the definition of the envelop (more generally, to include any kind of defective BC, such as the permeameter conditions which are clearly inappropriate for anisotropic media). With this restriction, the envelop is somewhat lower : the "safe region" becomes $\Lambda_{\text {Min }} /\langle\lambda\rangle \geq 1 / 3$, and the uncertainty can reach $0.02\langle\lambda\rangle / \Lambda_{M i n}$ out of this region.

In summary, $\Lambda_{\text {Min }} /\langle\lambda\rangle$ seems to provide an allpurpose indicator to detect situations that might be problematic in practical applications. Once a numerical upscaling has been performed by applying some particular $\mathrm{BC}, \Lambda_{\mathrm{Min}} /\langle\lambda\rangle$ is readily available and if it is too small, it is very likely that a solution of (1) with another BC would yield a significantly different result. Recall also that a noticeable asymmetry is also a worrying feature, since the asymmetry index $\mathscr{A}^{\prime}$ is a lower bound for the distance of $\boldsymbol{\Lambda}$ from any acceptable value (see Section II I). In case of doubts, appropriate measures should then be taken. The first step could be to try different BC's, and check whether the result is really impacted. If it is, the next step might be to consider ways to filter out the BC's influence, by excluding the potentially disturbed region from the measurement volume.

Aside from the identification of phenomenological features, the examination of the local fields in Section IV and especially the comparison of the fields resulting from different BC's aimed at the estimation of the depth from the boundaries reached by the BC's influence. The profiles of the RMS differences $\langle\widetilde{\delta T}\rangle_{2}$ in Figs. 9 and 10 provide an insight and show that the disturbed thickness is quite significant, of the order for all samples of at least 20 correlation lengths, including a steep drop in a shallow peripheral layer followed by an exponential decay with decay length $l_{d}$. However, this is not really conclusive, for several reasons. The ratios $l_{d} / l_{c}$ are different for the various samples (though of similar orders of magnitude), and sometimes even for different conductivity contrast in the same sample. The initial drop can be important or minute, and no rationalization was found to account for these differences. But most importantly, the value of $\langle\delta T\rangle_{2}$ at some position on the profile depends on the depth but also primarily on its value at sample boundary, which was not rationalized either, except of course for the fact that it increases with contrast.

A deeper analysis of this point will be conducted in a future work, where the use of synthetic media will make possible to vary systematically the geometrical parameters and get a broader picture than provided by the present tomographic images. However, the interest of this characterization is mainly to devise an a priori criterion to answer the question, "how large should a sample be to make sure that a central core exists where measurements unaffected by the kind of BC's can be made?" We cannot answer this yet, but nevertheless, given a specific sample, it is always possible to check whether such a core exists. This is the undersampling procedure described in Section $\mathrm{V}$, and it should be emphasized that it is virtually cost-free. Once problem (1) has been solved with any kind of $\mathrm{BC}$, measuring the mean flux $\langle\mathbf{q}\rangle_{c}$ and gradient $\langle\nabla T\rangle_{c}$ in a series of inner sub-domains does not require any significant additional computational effort.

Hence, we recommand the following protocol. When faced with a particular sample, if a first calculation with [BC1] yields $\boldsymbol{\Lambda}_{[B C 1]}$ with $\boldsymbol{\Lambda}_{M i n,[B C 1]} /\langle\lambda\rangle$ out of the "safe region", other calculations with other BC's should be made for a check. If the scatter of the results, quantified by their distances $\mathscr{D}^{\prime}$, exceeds what is regarded as tolerable for the targeted application, then the conductivity tensors $\boldsymbol{\Lambda}_{[B C], c}$ should be evaluated for the various BC's and for a series of inner domains. If $\boldsymbol{\Lambda}_{[B C], c}$ is found identical for all BC's and in a wide enough range of sub-domains sizes (within a userdefined tolerance for $\mathscr{D}^{\prime}$ ), this tensor can be regarded as an intrinsic effective property, associated with and only with the material under consideration. If this is not possible, it must be accepted that no reliable estimate of an effective conductivity tensor can be obtained from this sample.

Note that Fig.12 is incomplete in the sense that it only compares tensors $\Lambda_{[B C], c}$ from various BC's in the same subdomains, not their values in domains of different sizes. Conversely, Fig.11 compares the tensors for varying domain sizes, but only in terms of their mean diagonal. Both checks for the independance on the domain size and on the BC's have to be made in terms of the distance $\mathscr{D}^{\prime}$.

This search for a constant $\boldsymbol{\Lambda}_{[B C], c}$ can fail for two reasons: (i) because the predictions from the various BC's are never in satisfactory agreement; or (ii) because no range of domain size exist where these predictions remain acceptably constant (even if they are concordant). Success is possible only if the sample is large enough, so that the block after removal of a layer thick enough to screen the influence of the $\mathrm{BC}$ is still representative. Let us consider two examples taken from the foregoing.

Sample FS is an example of success but nearfailure for the second reason (ii). A threshold of $\pm 5 \%$ uncertainty was arbitrarily chosen, and the search was successful in the case of the most severe contrast $\lambda_{s} / \lambda_{g}=10^{-4}$ (and a fortiori for milder contrasts). In the range $35 \lesssim M \lesssim 115$ (remaining block size $232^{3} \sim 442^{3}$ ), all the tensors are found equal within $\pm 5 \%$ (green box in Fig. 11). Some values of $\mathscr{D}^{\prime}$ slightly larger than 0.10 are seen in Fig. 12 near $M=35$, but they involve the immersion condition [D/D], which we advocated to disregard. As a matter of fact, if $[\mathrm{D} / \mathrm{D}]$ is excluded, the predictions from all the BC's are found in very good agreement in this range $\left(\mathscr{D}^{\prime}\right.$ always $<$ 0.02 ), and the $\pm 5 \%$ uncertainty results for the most part from the dependance on the sampling domain size. This means that if the threshold were set at a smaller value, say $\pm 3 \%$, the search would fail because of the variations with the sampling domain size, even though 
the predictions from various BC's are concordant.

Of course, sample BP with a poorly conducting solid is an example of utter failure for both reasons (i) and (ii). The predictions from the various BC's are very different, even if $[D / D]$ is excluded $\left(\mathscr{D}^{\prime}\right.$ is $O(1)$ in Fig.12), and they strongly vary also with the sampling domain size. However, it is possible to come up with a fairly reliable conductivity (all values of $\mathscr{D}^{\prime} \leq 0.03$ ), without even resorting to the undersampling, when the contrast is mild or when the solid is very conducting $\left(\lambda_{s} / \lambda_{g} \geq 10^{-1}\right)$.
Let us conclude with this final remark. As already mentioned, investigations in future works involving a wider and more systematic exploration of geometric parameters, made possible by the use of synthetic stochatically generated samples, will hopefully help to rationalize the observations and improve the quantitative criteria introduced in Sections III and IV. But the self-diagnosing protocol proposed in the above and based on the undersampling procedure described in Section V applies in any case and can be used as it stands, regardless of any other consideration.
[1] Adler P.M., C. G. Jacquin \& J. A. Quiblier: Flow in simulated porous media. Int. J. Multiphase Flow, 16, 691$712(1990)$

[2] Adler P. M.: Porous Media: Geometry and Transports. Butterworth/Heinemann, Stoneham, MA. (1992)

[3] Alam K., M.S. Anghelescu \& Adrian Bradu: Computational Model of Porous Media Using True 3-D Images. In: Öchsner A. \& G.E. Murch (eds.), Heat Transfer in Multi-Phase Materials, Adv Struct Mater 2, DOI 10.1007/8611_2010_7, Springer-Verlag Berlin Heidelberg (2010)

[4] Andrä H., N. Combaret, J. Dvorkin, E. Glatt, J. Han, M. Kabel, Y. Keehm, F. Krzikalla, M. Lee, C. Madonna, M. Marsh, T. Mukerji, E.H. Saenger, R. Sain, N. Saxena, S. Ricker, A. Wiegmann \& , X. Zhan: Digital rock physics benchmarks - part II: Computing effective properties. Computers \& Geosciences, 50, 33-43 (2013)

[5] Bailly D., R. Ababou \& M. Quintard: Geometric characterization, hydraulic behavior and upscaling of 3D fissured geologic media. Mathematics and Computers in Simulation, 79, 3385-3396 (2009)

[6] Bailly D.: Vers une modélisation des écoulements dans les massifs très fissurés de type karst : étude morphologique, hydraulique et changement d'échelle. $\mathrm{PhD}$ Thesis, Université de Toulouse (2009)

[7] Blunt M.J., B. Bijeljic, H. Dong, O. Gharbi, S. Iglauer, P. Mostaghimi, A. Paluszny \& C. Pentland: Pore-scale imaging and modelling. Advances in Water Resources, 51, 197-216 (2013)

[8] Bruggeman, D. A. G.: Berechnung verschiedener physikalischer konstanten von heterogenen substanzen. Annalen der Physik, 24, 636-679 (1935)

[9] De Lucia M., C.1 de Fouquet, V. Lagneau \& Roberto Bruno: Equivalent block transmissivity in an irregular 2D polygonal grid for one-phase flow: A sensitivity analysis. C. R. Geoscience, 341 327-338 (2009)

[10] Durlofsky L.J.: Numerical Calculation of Equivalent Grid Block Permeability Tensors for Heterogeneous Porous Media. Water Resours. Res., 27, 699-708 (1991)

[11] Durlofsky L.J.: Upscaling and Gridding of Fine Scale Geological Models for Flow Simulation. 8th International Forum on Reservoir Simulation, Iles Borromees, Stresa, Italy (2005)

[12] Ferry L. : Caractérisation de résidus de combustion de câbles électriques, Technical report PTF18-30/LAF, IMT Mines Alès (2018)

[13] Gomez-Hermindez, J. J. \& A.G. Journel: Stochastic characterization of grid-block permeabilities: from point values to block tensors. In 2nd European Con- ference on the Mathematics of Oil Recovery, eds. D. Guerillot and 0. Guillon. Edition Technip, Paris, pp. 8390 (1990)

[14] Guibert R., P. Horgue, G. Debenest \& M. Quintard: A Comparison of Various Methods for the Numerical Evaluation of Porous Media Permeability Tensors from Pore-Scale Geometry. Math Geosci, 48, 329-347 (2016)

[15] Hashin Z. and S. Shtrikman: A variational approach to the theory of the effective magnetic permeability of multiphase materials, J. Appl. Phys., 33, 3125-3131 (1962)

[16] Haussener S., P. Coray, W. Lipinski, P. Wyss \& A. Steinfeld: Tomography-Based Heat and Mass Transfer Characterization of Reticulate Porous Ceramics for HighTemperature Processing. J. Heat Transfer, 132, 023305 (2010)

[17] Henriette A., C.G. Jacquin, P.M. Adler: The effective permeability of heterogeneous porous media. Phys. Chem. Hydrodynamics, 11, 63-80 (1989)

[18] Khan F., F. Enzmann, M. Kersten, A. Wiegmann \& K. Steiner: 3D simulation of the permeability tensor in a soil aggregate on basis of nanotomographic imaging and LBE solver. Soils and Sediments, 12, 86-96 (2012)

[19] Landauer R.: Electrical conductivity in inhomogeneous media. AIP Conference Proceedings, 40, 2-43 (1978)

[20] Lang P. S., A. Paluszny \& R. W. Zimmerman: Permeability tensor of three-dimensional fractured porous rock and a comparison to trace map predictions. J. Geophys. Res. Solid Earth, 119, 6288-6307 (2014)

[21] Long J.C.S., J.S. Remer, C.R. Wilson \& P.A. Witherspoon: Porous Media Equivalents for Networks of Discontinuous Fractures. Water Resours. Res, 18, 645-658 (1982)

[22] Mostaghimi P., M.J. Blunt \& B. Bijeljic: Computations of Absolute Permeability on Micro-CT Images. Math Geosci, 45, 103-125 (2013)

[23] Petrasch J., B. Schrader, P. Wyss \& A. Steinfeld: Tomography-Based Determination of the Effective Thermal Conductivity of Fluid-Saturated Reticulate Porous Ceramics. J. Heat Transfer, 130, 032602 (2008)

[24] Petrasch J., F. Meier, H. Friess \& A. Steinfeld: Tomography based determination of permeability, Dupuit-Forchheimer coefficient, and interfacial heat transfer coefficient in reticulate porous ceramics. Int. J. of Heat and Fluid Flow, 29, 315-326 (2008)

[25] Piller M., G. Schena, M. Nolich, S. Favretto, F. Radaelli \& E. Rossi: Analysis of Hydraulic Permeability in Porous Media: From High Resolution X-ray Tomography to Direct Numerical Simulation. Transp Porous 
Med 80, 57-78 (2009)

[26] Pouya A. \& O. Fouché: Permeability of 3D discontinuity networks: New tensors from boundary-conditioned homogenisation. Advances in Water Resources, 32, 303-314 (2009)

[27] Ralston, A. \& P.A. Rabinowitz : First Course in Numerical Analysis, Dover Publications, Mineola, N.Y. (2001)

[28] Renard P. \& G.de Marsily: Calculating equivalent permeability: a review. Advances in Water Resources, 20, 253-278 (1997)

[29] Shi J., G. Boyer \& J.-F. Thovert: Simulation of the pyrolysis of charring polymers: influence of the porous media properties. Proc. European Symposium of Fire Safety Science, ESFSS 2018, Nancy, France (2018)

[30] Spanne P., J.-F. Thovert, C.J. Jacquin, W.B. Lindquist, K.W. Jones \& P.M. Adler: Synchrotron computed microtomography of porous media. Topology and transports, Phys. Rev. Lett., 73, 2001-2004 (1994)

[31] Thovert J.-F., F. Yousefian, P. Spanne, C.G. Jacquin \& P.M. Adler: Grain reconstruction of porous media: application to a low-porosity Fontainebleau sandstone. Phys. Rev. E, 63, 61307-61323 (2001)

[32] Thovert J.-F. \& P.M. Adler: Grain reconstruction of porous media: Application to a Bentheim sandstone. Phys. Rev E, 83, 056116 (2011)
[33] Wen, X.H., L. J. Durlofsk \& M. G. Edwards: Use of Border Regions for Improved Permeability Upscaling, Mathematical Geology, 35, 521-547 (2003)

[34] Wiener O.: Die Theorie des Mischkörpers für das Feld des stationären Strömung. Erste Abhandlung: Die Mittelwertsätze für Kraft, Polarisation und Energie, $A b$ handl. d.K.S. Gesellsch.d. Wisseensch. Math.-Phys., 32, 509-604 (1912)

[35] Wu X., T. Hou \& Y. Efendiev: Analysis of upscaling absolute permeability. Discrete Contin. Dyn. Syst. Ser. B, 2, 185-204 (2002)

\section{ACKNOWLEDGEMENTS}

We gratefully thank Laurent Ferry (C2MA) for preparing the samples of thermally degraded polymers, and Pascal Laheurte (LEM3) for providing their tomographic images. This work pertains to the French Government Programme "Investissements d'Avenir" (LABEX INTERACTIFS, reference ANR-11-LABX0017-01). 